\title{
Oxidation Reactions of a Series of Benzidines. Electrochemical \\ Detection of Dimerization of Cation Radicals and Examples of \\ Potential Inversion Instigated by Very Small Steric Effects
}

Norma A. Macías-Ruvalcaba and Dennis H. Evans*

Department of Chemistry, University of Arizona, Tucson, AZ 85721

(USA)

\section{Supporting Information}

1. Figures S1 and S2. Temperature-dependent spectra of cation radical salts of $\mathbf{1 b}$ and $\mathbf{1 c}$.

2. Figures S3-S5. Apparent molar absorptivity vs. total concentration of cation radical salts of $\mathbf{1 b}$-d.

3. Tables S1-S10. Simulation parameter values used to fit voltammograms for $\mathbf{1 a - d}, \mathbf{1 j}, \mathbf{1 e - h}$ and $\mathbf{1 k}$, respectively.

4. Comment on the magnitude of dimerization rate constants,

5. Figures S6-S21. Additional examples of fits of simulation to experimental voltammograms for 1a-d, 1j, and 1e-h. 


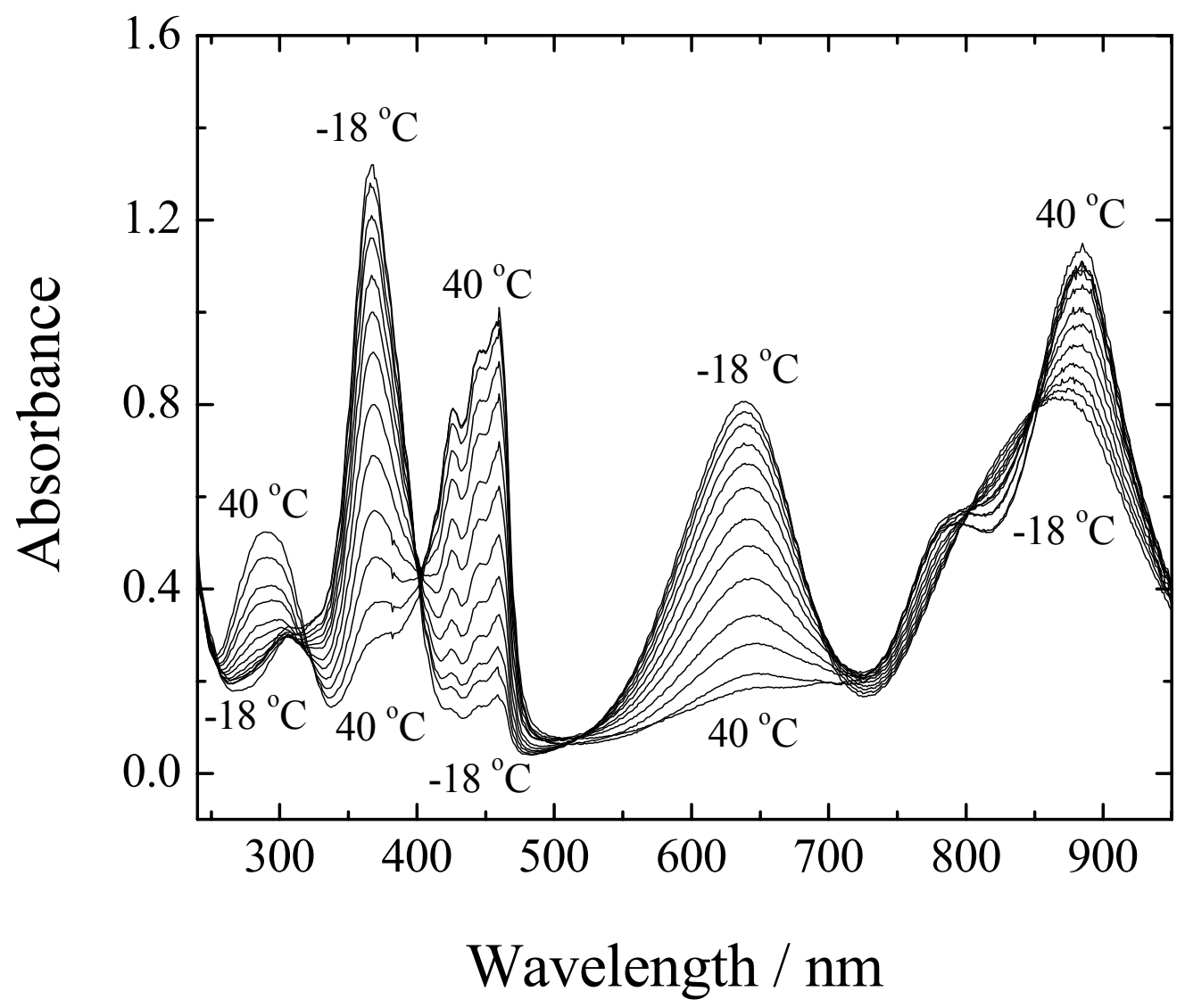

Figure S1. Temperature dependence of the absorption spectrum of $4.3 \times 10^{-5} \mathrm{M}^{1} \mathbf{b}^{\mathbf{}} \mathrm{ClO}_{4}{ }^{-}$ in $0.1 \mathrm{M} \mathrm{Bu}_{4} \mathrm{NPF}_{6}$ in acetonitrile. Temperatures: $-18,-15,-10,-5,0,5,10,15,20,25,30$, $35,40{ }^{\circ} \mathrm{C}$. 


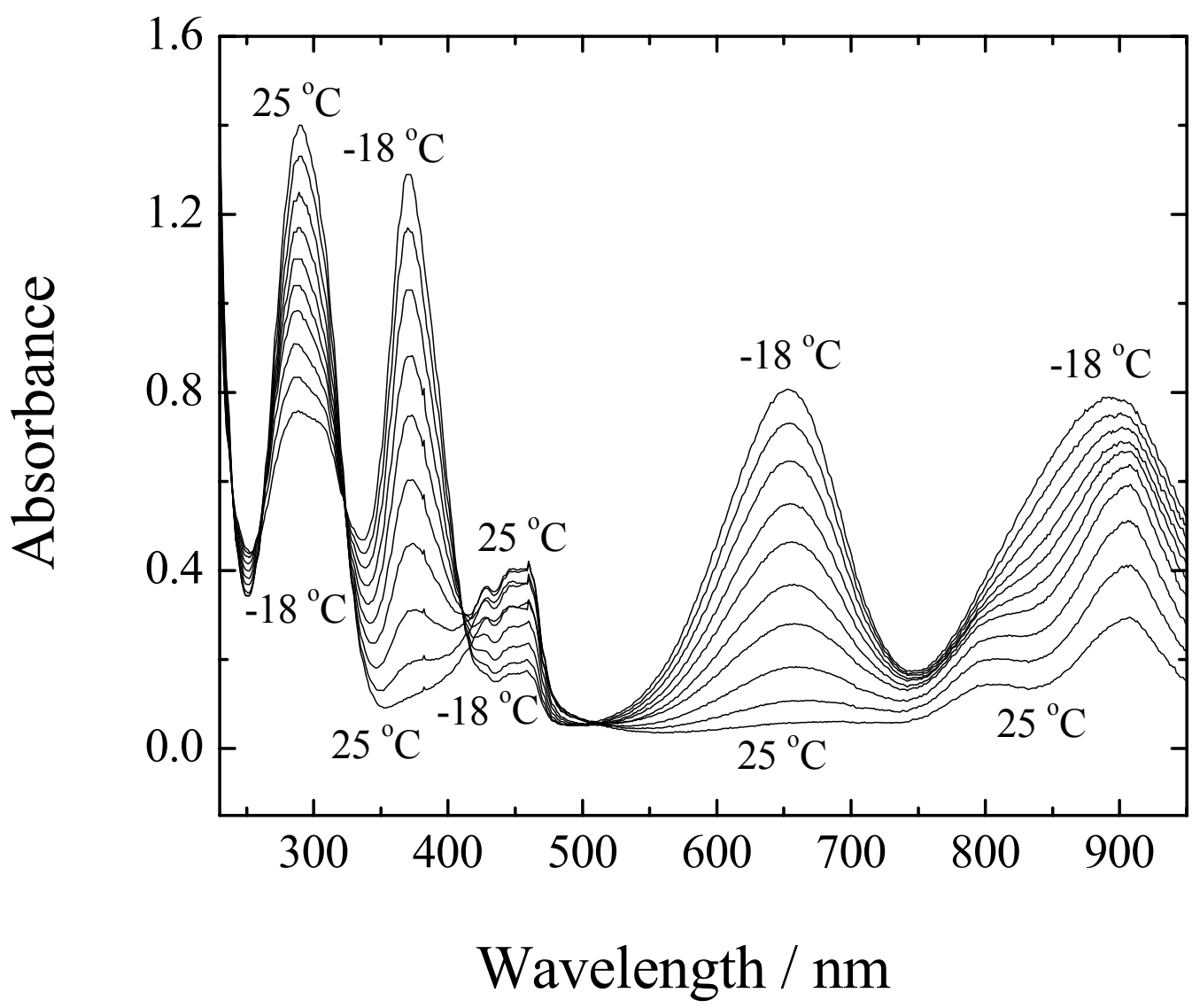

Figure S2. Temperature dependence of the absorption spectrum of $5.88 \times 10^{-5} \mathrm{M}$ $1 \mathbf{c}^{\circ+} \mathrm{ClO}_{4}^{-}$in $0.1 \mathrm{M} \mathrm{Bu}_{4} \mathrm{NPF}_{6}$ in acetonitrile. Temperatures: $-18,-15,-10,-5,0,5,10,15$, $20,25^{\circ} \mathrm{C}$. 


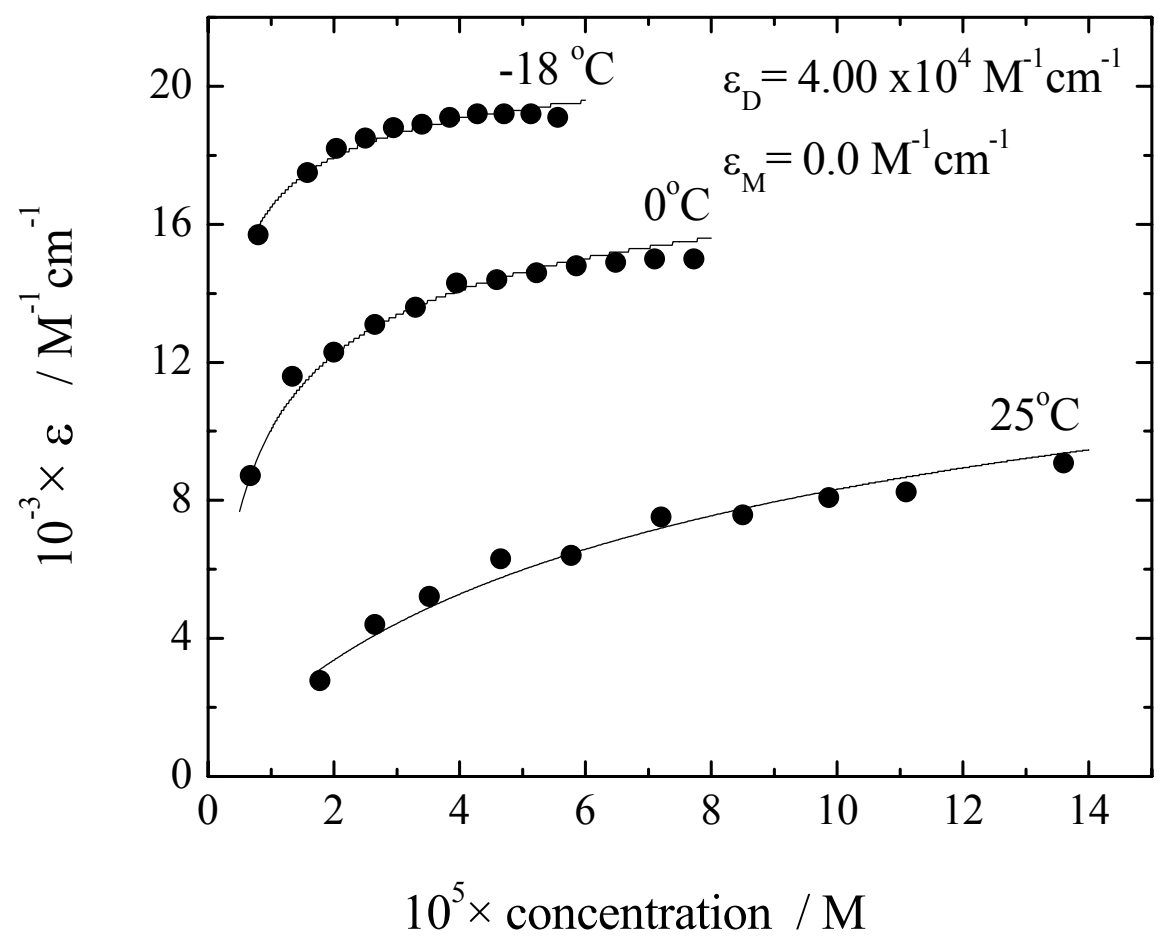

Figure S3. Apparent molar absorptivity (absorbance/total concentration of $1 \mathbf{b}^{\bullet+} \mathrm{ClO}_{4}^{-}$) at $646 \mathrm{~nm}$ for various concentrations of cation radical for three different temperatures (filled circles). Full curves: fits of the data according to equation 1 with molar absorptivities as shown and $K_{\text {dim }}=6.10 \times 10^{3} \mathrm{M}^{-1}, 1.10 \times$ $10^{5} \mathrm{M}^{-1}$ and $5.94 \times 10^{5} \mathrm{M}^{-1}$ for 25,0 and $-18^{\circ} \mathrm{C}$, respectively. 


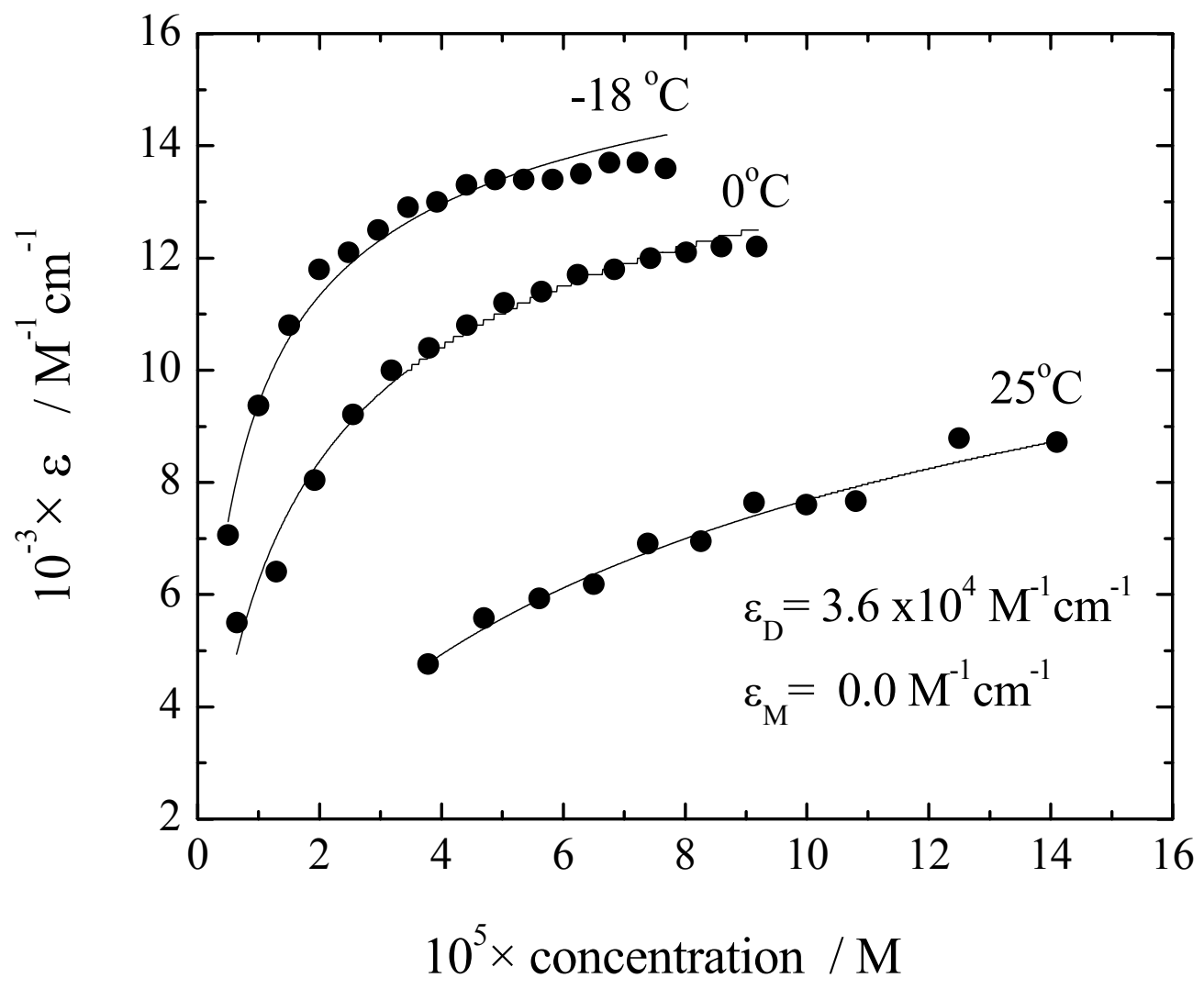

Figure S4. Apparent molar absorptivity (absorbance/total concentration of $1 \mathbf{c}^{\circ+} \mathrm{ClO}_{4}{ }^{-}$) at $660 \mathrm{~nm}$ for various concentrations of cation radical for three different temperatures (filled circles). Full curves: fits of the data according to equation 1 with molar absorptivities as shown and $K_{\mathrm{dim}}=6.50 \times 10^{3} \mathrm{M}^{-1}, 4.07 \times$ $10^{4} \mathrm{M}^{-1}$ and $1.15 \times 10^{5} \mathrm{M}^{-1}$ for 25,0 and $-18{ }^{\circ} \mathrm{C}$, respectively. 


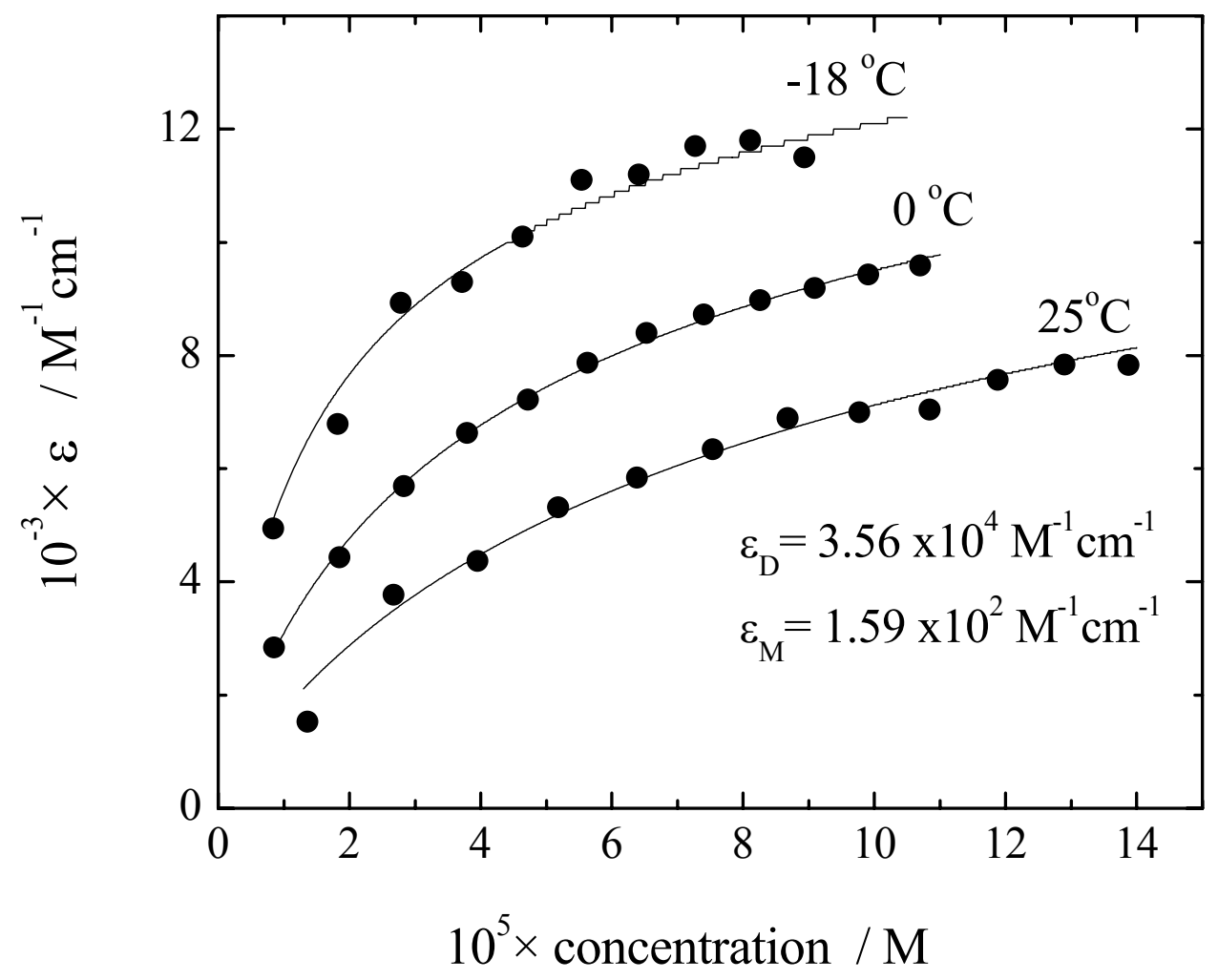

Figure S5. Apparent molar absorptivity (absorbance/total concentration of $\mathbf{1 d}^{\bullet+} \mathrm{ClO}_{4}{ }^{-}$) at $661 \mathrm{~nm}$ for various concentrations of cation radical for three different temperatures (filled circles). Full curves: fits of the data according to equation 1 with molar absorptivities as shown and $K_{\text {dim }}=5.37 \times 10^{3} \mathrm{M}^{-1}, 1.19 \times$ $10^{4} \mathrm{M}^{-1}$ and $3.22 \times 10^{4} \mathrm{M}^{-1}$ for 25,0 and $-18^{\circ} \mathrm{C}$, respectively.

Tables S1-S10. Generic footnote a: ${ }^{a}$ Potentials are expressed in V vs. ferrocenium/ferrocene. Units for $k_{\mathrm{s}}: \mathrm{cm} / \mathrm{s}$; equilibrium constants are for $1 \mathrm{M}$ standard state; units for first-order rate constants: $\mathrm{s}^{-1}$; second-order rate constants: $\mathrm{M}^{-1} \mathrm{~s}^{-1}$. The same values were used to fit all scan rates from 0.1 to $30.0 \mathrm{~V} / \mathrm{s}$. Resistance compensation. Format: Temperature $\left({ }^{\circ} \mathrm{C}\right) /$ Total resistance $(\Omega) /$ Resistance electronically compensated $(\Omega): 25 / 140 / 120 ; 0 / 190 / 130 ;-18 / 220 / 200$. The residual uncompensated resistance was included in the simulations. 
Table S1. Experimental conditions and simulation parameters for $o$-dianisidine, 1a, in acetonitrile. ${ }^{\text {a }}$

\begin{tabular}{lcccccc}
\hline Concentration/ Temp. ${ }^{\circ} \mathrm{C}$ & \multicolumn{2}{c}{$1.75 \mathrm{mM}$ 1a $/ 25^{\circ} \mathrm{C}$} & \multicolumn{3}{c}{$3.70 \mathrm{mM} 1 \mathbf{a} / 25{ }^{\circ} \mathrm{C}$} \\
\hline Electrochemical reactions & $E^{\circ}$ & $\alpha$ & $k_{s}$ & $E^{\circ}$ & $\alpha$ & $k_{s}$ \\
\hline $\mathrm{A}^{+}+\mathrm{e}=\mathrm{A}$ & 0.092 & 0.50 & 0.50 & 0.092 & 0.50 & 0.50 \\
$\mathrm{~A}^{2+}+\mathrm{e}=\mathrm{A}^{+}$ & 0.240 & 0.65 & 0.40 & 0.242 & 0.65 & 0.40 \\
\hline Chemical reactions $^{+}$ & $K$ & $k_{f}$ & $k_{b}$ & $K$ & $k_{f}$ & $k_{b}$ \\
\hline $\mathrm{A}^{+}+\mathrm{A}^{+}=\mathrm{AA}^{2+}$ & $1.8 \times 10^{3}$ & $7.0 \times 10^{7}$ & $4.0 \times 10^{4}$ & $1.8 \times 10^{3}$ & $7.0 \times 10^{7}$ & $4.0 \times 10^{4}$ \\
$\mathrm{~A}^{+}+\mathrm{A}^{+}=\mathrm{A}+\mathrm{A}^{2+}$ & $3.2 \times 10^{-3}$ & $3.5 \times 10^{2}$ & $1.1 \times 10^{5}$ & $3.0 \times 10^{-3}$ & $3.5 \times 10^{2}$ & $1.2 \times 10^{5}$ \\
$\mathrm{~A}^{2+}=\mathrm{Prod}^{\mathrm{b}}$ & $1.0 \times 10^{9}$ & 0.26 & $2.6 \times 10^{-10}$ & $1.0 \times 10^{9}$ & 0.26 & $2.6 \times 10^{-10}$ \\
$D_{\mathrm{A}} ; D_{\mathrm{A}+, \mathrm{A} 2+, \mathrm{AA} 2+} / \mathrm{cm}^{2} \mathrm{~s}^{-1}$ & \multicolumn{2}{c}{$1.46 \times 10^{-5} ; 1.05 \times 10^{-5}$} & & $1.51 \times 10^{-5} ; 1.00 \times 10^{-5}$ \\
\hline
\end{tabular}

Concentration/ Temp. ${ }^{\circ} \mathrm{C} \quad 1.30 \mathrm{mM} \mathrm{1a} / 0{ }^{\circ} \mathrm{C}$

$3.28 \mathrm{mM} \mathrm{1a} / 0{ }^{\circ} \mathrm{C}$

\begin{tabular}{lcccccc} 
Electrochemical reactions & $E^{\circ}$ & $\alpha$ & $k_{s}$ & $E^{\circ}$ & $\alpha$ & $k_{s}$ \\
\hline $\mathrm{A}^{+}+\mathrm{e}=\mathrm{A}$ & 0.086 & 0.50 & 0.48 & 0.084 & 0.50 & 0.48 \\
$\mathrm{~A}^{2+}+\mathrm{e}=\mathrm{A}^{+}$ & 0.224 & 0.63 & 0.35 & 0.221 & 0.63 & 0.35
\end{tabular}

Chemical reactions

K

$k_{f} \quad k_{b}$

K

$k_{f}$

$k_{b}$

\begin{tabular}{lcccccc}
$\mathrm{A}^{+}+\mathrm{A}^{+}=\mathrm{AA}^{2+}$ & $1.4 \times 10^{4}$ & $6.0 \times 10^{8}$ & $4.4 \times 10^{4}$ & $1.4 \times 10^{4}$ & $6.0 \times 10^{8}$ & $4.4 \times 10^{4}$ \\
$\mathrm{~A}^{+}+\mathrm{A}^{+}=\mathrm{A}+\mathrm{A}^{2+}$ & $2.9 \times 10^{-3}$ & $1.2 \times 10^{3}$ & $4.2 \times 10^{5}$ & $2.9 \times 10^{-3}$ & $1.2 \times 10^{3}$ & $4.2 \times 10^{5}$ \\
$\mathrm{~A}^{2+}=\mathrm{Prod}^{\mathrm{b}}$ & $1.0 \times 10^{9}$ & 0.26 & $2.6 \times 10^{-10}$ & $1.0 \times 10^{9}$ & 0.26 & $2.6 \times 10^{-10}$ \\
$D_{\mathrm{A}, \mathrm{A}+, \mathrm{A} 2+} ; D_{\mathrm{AA} 2+} / \mathrm{cm}^{2} \mathrm{~s}^{-1}$ & \multicolumn{2}{c}{$1.05 \times 10^{-5} ; 7.54 \times 10^{-6}$} & $1.10 \times 10^{-5} ; 7.65 \times 10^{-6}$ \\
\hline
\end{tabular}

Concentration/ Temp. ${ }^{\circ} \mathrm{C}$

$0.95 \mathrm{mM} \mathrm{1a} /-18^{\circ} \mathrm{C}$

$3.80 \mathrm{mM} \mathrm{1a} /-18^{\circ} \mathrm{C}$

\begin{tabular}{lcccccc} 
Electrochemical reactions & $E^{\circ}$ & $\alpha$ & $k_{s}$ & $E^{\circ}$ & $\alpha$ & $k_{s}$ \\
$\mathrm{~A}^{+}+\mathrm{e}=\mathrm{A}$ & 0.090 & 0.50 & 0.20 & 0.085 & 0.50 & 0.20 \\
$\mathrm{~A}^{2+}+\mathrm{e}=\mathrm{A}^{+}$ & 0.216 & 0.66 & 0.32 & 0.209 & 0.66 & 0.32 \\
\hline Chemical reactions & $K$ & $k_{f}$ & $k_{b}$ & $K$ & $k_{f}$ & $k_{b}$ \\
\hline $\mathrm{A}^{+}+\mathrm{A}^{+}=\mathrm{AA}^{2+}$ & $8.5 \times 10^{4}$ & $1.7 \times 10^{9}$ & $2.0 \times 10^{4}$ & $8.5 \times 10^{4}$ & $1.7 \times 10^{9}$ & $2.0 \times 10^{4}$ \\
$\mathrm{~A}^{+}+\mathrm{A}^{+}=\mathrm{A}+\mathrm{A}^{2+}$ & $3.3 \times 10^{-3}$ & $3.3 \times 10^{2}$ & $1.0 \times 10^{5}$ & $3.6 \times 10^{-3}$ & $3.3 \times 10^{2}$ & $9.2 \times 10^{4}$ \\
$\mathrm{~A}^{2+}=\mathrm{Prod}^{\mathrm{b}}$ & $1.0 \times 10^{9}$ & 0.24 & $2.4 \times 10^{-10}$ & $1.0 \times 10^{9}$ & 0.24 & $2.4 \times 10^{-10}$ \\
$D_{\mathrm{A}, \mathrm{A}+, \mathrm{A} 2+} ; D_{\mathrm{AA} 2+} / \mathrm{cm}^{2} \mathrm{~s}^{-1}$ & \multicolumn{2}{c}{$8.46 \times 10^{-6} ; 5.46 \times 10^{-6}$} & \multicolumn{2}{c}{$8.70 \times 10^{-6} ; 6.15 \times 10^{-6}$}
\end{tabular}

${ }^{\mathrm{b}}$ Slow decompostion of dication improved fits. 
Table S2. Experimental conditions and simulation parameters for $\boldsymbol{o}$-tolidine, $\mathbf{1 b}$, in acetonitrile. ${ }^{\mathrm{a}}$

\begin{tabular}{lcccccc}
\hline Concentration/ Temp. ${ }^{\circ} \mathrm{C}$ & \multicolumn{2}{c}{$1.15 \mathrm{mM}$ 1b $/ 25^{\circ} \mathrm{C}$} & \multicolumn{3}{c}{$3.52 \mathrm{mM} \mathbf{1 b} / 25{ }^{\circ} \mathrm{C}$} \\
\hline Electrochemical reactions & $E^{\circ}$ & $\alpha$ & $k_{s}$ & $E^{\circ}$ & $\alpha$ & $k_{s}$ \\
\hline $\mathrm{A}^{+}+\mathrm{e}=\mathrm{A}$ & 0.136 & 0.50 & 0.41 & 0.135 & 0.50 & 0.41 \\
$\mathrm{~A}^{2+}+\mathrm{e}=\mathrm{A}^{+}$ & 0.304 & 0.50 & 0.25 & 0.304 & 0.50 & 0.25 \\
\hline Chemical reactions & $K$ & $k_{f}$ & $k_{b}$ & $K$ & $k_{f}$ & $k_{b}$ \\
\hline $\mathrm{A}^{+}+\mathrm{A}^{+}=\mathrm{AA}^{2+}$ & $6.0 \times 10^{3}$ & $1.3 \times 10^{8}$ & $2.2 \times 10^{4}$ & $6.0 \times 10^{3}$ & $1.3 \times 10^{8}$ & $2.2 \times 10^{4}$ \\
$\mathrm{~A}^{+}+\mathrm{A}^{+}=\mathrm{A}+\mathrm{A}^{2+}$ & $1.4 \times 10^{-3}$ & $9.2 \times 10^{2}$ & $6.4 \times 10^{5}$ & $1.4 \times 10^{-3}$ & $9.2 \times 10^{2}$ & $6.7 \times 10^{5}$ \\
$\mathrm{~A}^{2+}=\mathrm{Prod}^{\mathrm{b}}$ & $1.0 \times 10^{9}$ & 0.20 & $2.0 \times 10^{-10}$ & $1.0 \times 10^{9}$ & 0.20 & $2.0 \times 10^{-10}$ \\
$D_{\mathrm{A}} ; D_{\mathrm{A}+, \mathrm{A} 2+, \mathrm{AA} 2+} / \mathrm{cm}^{2} \mathrm{~s}^{-1}$ & \multicolumn{2}{c}{$1.38 \times 10^{-5} ; 1.14 \times 10^{-5}$} & & $1.50 \times 10^{-5} ; 1.20 \times 10^{-5}$ \\
\hline
\end{tabular}

Concentration/ Temp. ${ }^{\circ} \mathrm{C} \quad 1.30 \mathrm{mM} \mathrm{1b} / 0{ }^{\circ} \mathrm{C}$

$3.38 \mathrm{mM} \mathrm{1b} / 0{ }^{\circ} \mathrm{C}$

\begin{tabular}{lcccccc} 
Electrochemical reactions & $E^{\circ}$ & $\alpha$ & $k_{s}$ & $E^{\circ}$ & $\alpha$ & $k_{s}$ \\
\hline $\mathrm{A}^{+}+\mathrm{e}=\mathrm{A}$ & 0.124 & 0.50 & 0.21 & 0.123 & 0.50 & 0.21 \\
$\mathrm{~A}^{2+}+\mathrm{e}=\mathrm{A}^{+}$ & 0.278 & 0.62 & 0.21 & 0.278 & 0.62 & 0.21 \\
\hline
\end{tabular}

Chemical reactions

$\begin{array}{lll}K & k_{f} & k_{b}\end{array}$

$\mathrm{A}^{+}+\mathrm{A}^{+}=\mathrm{AA}^{2+}$

$1.1 \times 10^{5} \quad 1.6 \times 10^{9}$

$1.5 \times 10^{4}$

K

$k_{f}$

$k_{b}$

$\mathrm{A}^{+}+\mathrm{A}^{+}=\mathrm{A}+\mathrm{A}^{2+}$

$1.4 \times 10^{-3} \quad 3.4 \times 10^{2}$

$2.4 \times 10^{5}$

$1.1 \times 10^{5}$

$1.6 \times 10^{9}$

$1.5 \times 10^{4}$

$\mathrm{A}^{2+}=\operatorname{Prod}^{\mathrm{b}}$

$1.0 \times 10^{9}$

0.17

$1.7 \times 10^{-10}$

$1.4 \times 10^{-3} \quad 3.4 \times 10^{2}$

$2.4 \times 10^{5}$

$D_{\mathrm{A}, \mathrm{A}+, \mathrm{A} 2+} ; D_{\mathrm{AA} 2+} / \mathrm{cm}^{2} \mathrm{~s}^{-1}$

$1.03 \times 10^{-5} ; 6.00 \times 10^{-6}$

$1.0 \times 10^{9}$

0.17

$1.7 \times 10^{-10}$

Concentration/ Temp. ${ }^{\circ} \mathrm{C}$

$1.10 \mathrm{mM} 1 \mathrm{~b} /-18^{\circ} \mathrm{C}$

$3.52 \mathrm{mM} \mathbf{1 b} /-18^{\circ} \mathrm{C}$

Electrochemical reactions

$E^{\circ}$

$\mathrm{A}^{+}+\mathrm{e}=\mathrm{A}$

0.129

$\alpha \quad k_{s}$

$1.04 \times 10^{-5} ; 6.15 \times 10^{-6}$

$\mathrm{A}^{2+}+\mathrm{e}=\mathrm{A}^{+}$

0.273

0.50

0.17

$E^{\circ}$

$\alpha$ $k_{s}$

Chemical reactions

K

0.56

0.21

0.125

0.50

0.17

$\mathrm{A}^{+}+\mathrm{A}^{+}=\mathrm{AA}^{2+}$

$5.9 \times 10^{5}$

$k_{f}$

$k_{b}$

0.265

0.56

0.21

$\mathrm{A}^{+}+\mathrm{A}^{+}=\mathrm{A}+\mathrm{A}^{2+}$

$1.4 \times 10^{-3}$

$2.3 \times 10^{10}$

$3.9 \times 10^{4}$

$K$

$k_{f}$

$k_{b}$

$\mathrm{A}^{2+}=\operatorname{Prod}^{\mathrm{b}}$

$1.0 \times 10^{9}$

$1.0 \times 10^{3}$

$7.2 \times 10^{5}$

$5.9 \times 10^{5}$

$2.3 \times 10^{10}$

$3.9 \times 10^{4}$

$D_{\mathrm{A}, \mathrm{A}+, \mathrm{A} 2+} ; D_{\mathrm{AA} 2+} / \mathrm{cm}^{2} \mathrm{~s}^{-1}$

0.30

$3.0 \times 10^{-10}$

$1.8 \times 10^{-3}$

$1.0 \times 10^{3}$

$5.6 \times 10^{5}$

$8.68 \times 10^{-6} ; 4.70 \times 10^{-6}$

$1.0 \times 10^{9}$

0.30

$3.0 \times 10^{-10}$

${ }^{\mathrm{b}}$ Slow decompostion of dication improved fits. 
Table S3. Experimental conditions and simulation parameters for 3,3'-dichlorobenzidine, 1c, in acetonitrile. ${ }^{\mathrm{a}}$

Concentration/ Temp. ${ }^{\circ} \mathrm{C} \quad 2.00 \mathrm{mM} \mathrm{1c} / 25^{\circ} \mathrm{C}$

$4.49 \mathrm{mM} 1 \mathrm{c} / 25^{\circ} \mathrm{C}$

Electrochemical reactions $\quad E^{\circ} \quad \alpha \quad k_{s} \quad E^{\circ} \quad \alpha \quad \alpha \quad k_{s}$

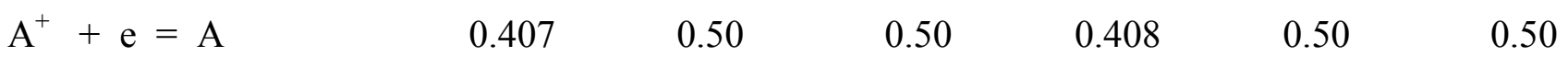

$\mathrm{A}^{2+}+\mathrm{e}=\mathrm{A}^{+}$

0.559

0.70

0.39

0.559

0.70

0.39

Chemical reactions

K

$k_{f} \quad k_{b}$

K

$k_{f}$
$k_{b}$
$\mathrm{A}^{+}+\mathrm{A}^{+}=\mathrm{AA}^{2+}$
$6.5 \times 10^{3} \quad 1.0 \times 10^{9}$
$1.5 \times 10^{5}$
$6.5 \times 10^{3}$
$1.0 \times 10^{9}$
$1.5 \times 10^{5}$
$\mathrm{A}^{+}+\mathrm{A}^{+}=\mathrm{A}+\mathrm{A}^{2+}$
$2.8 \times 10^{-3} \quad 1.0 \times 10^{5}$
$3.6 \times 10^{7}$
$2.9 \times 10^{-3} \quad 1.0 \times 10^{5}$
$3.5 \times 10^{7}$
$\mathrm{A}^{2+}=\operatorname{Prod}^{\mathrm{b}}$
$1.0 \times 10^{9}$
0.17
$1.7 \times 10^{-10}$
$1.0 \times 10^{9}$
0.17
$1.7 \times 10^{-10}$

$D_{\mathrm{A}} ; D_{\mathrm{A}+, \mathrm{A} 2+, \mathrm{AA} 2+} / \mathrm{cm}^{2} \mathrm{~s}^{-1}$

$1.68 \times 10^{-5} ; 1.15 \times 10^{-5}$

$1.48 \times 10^{-5} ; 1.15 \times 10^{-5}$

Concentration/ Temp. ${ }^{\circ} \mathrm{C} \quad 1.22 \mathrm{mM} \mathrm{1c} / 0{ }^{\circ} \mathrm{C}$

$3.30 \mathrm{mM} \mathrm{1c} / 0{ }^{\circ} \mathrm{C}$

\begin{tabular}{lcccccc} 
Electrochemical reactions & $E^{\circ}$ & $\alpha$ & $k_{s}$ & $E^{\circ}$ & $\alpha$ & $k_{s}$ \\
\hline $\mathrm{A}^{+}+\mathrm{e}=\mathrm{A}$ & 0.393 & 0.50 & 0.42 & 0.391 & 0.50 & 0.42 \\
$\mathrm{~A}^{2+}+\mathrm{e}=\mathrm{A}^{+}$ & 0.548 & 0.60 & 0.18 & 0.548 & 0.60 & 0.17 \\
\hline
\end{tabular}

Chemical reactions

K

$k_{f} \quad k_{b}$

K

$k_{f}$

$k_{b}$

$\mathrm{A}^{+}+\mathrm{A}^{+}=\mathrm{AA}^{2+}$

$4.1 \times 10^{4} \quad 7.0 \times 10^{9}$

$1.7 \times 10^{5}$

$4.1 \times 10^{4}$

$7.0 \times 10^{9}$

$1.7 \times 10^{5}$

$\mathrm{A}^{+}+\mathrm{A}^{+}=\mathrm{A}+\mathrm{A}^{2+}$

$1.4 \times 10^{-3}$

$3.4 \times 10^{2}$

$2.5 \times 10^{5}$

$1.3 \times 10^{-3}$

$3.4 \times 10^{2}$

$2.7 \times 10^{5}$

$\mathrm{A}^{2+}=\operatorname{Prod}^{\mathrm{b}}$

$1.0 \times 10^{9}$

0.21

$2.1 \times 10^{-10}$

$1.0 \times 10^{9}$

0.21

$2.1 \times 10^{-10}$

$D_{\mathrm{A}, \mathrm{A}+, \mathrm{A} 2+} ; D_{\mathrm{AA}^{2}+} / \mathrm{cm}^{2} \mathrm{~s}^{-1}$

$1.13 \times 10^{-5} ; 7.20 \times 10^{-6}$

$1.20 \times 10^{-5} ; 7.20 \times 10^{-6}$

Concentration/ Temp. ${ }^{\circ} \mathrm{C}$

$1.54 \mathrm{mM} 1 \mathrm{c} /-18^{\circ} \mathrm{C}$

$3.27 \mathrm{mM} \mathrm{1c} /-18^{\circ} \mathrm{C}$

Electrochemical reactions

$E^{\circ}$

$\alpha \quad k_{s}$

$E^{\circ}$

$\alpha$

$k_{s}$

$\mathrm{A}^{+}+\mathrm{e}=\mathrm{A}$

0.379

0.50

0.16

0.381

0.50

0.16

$\mathrm{A}^{2+}+\mathrm{e}=\mathrm{A}^{+}$

0.542

0.60

0.18

0.542

0.60

0.18

Chemical reactions

K

$k_{f}$

$k_{b}$

K

$k_{f}$ $k_{b}$

$\mathrm{A}^{+}+\mathrm{A}^{+}=\mathrm{AA}^{2+}$

$1.2 \times 10^{5} \quad 5.0 \times 10^{9}$

$4.3 \times 10^{4}$

$1.2 \times 10^{5}$

$5.0 \times 10^{9}$

$4.3 \times 10^{4}$

$\mathrm{A}^{+}+\mathrm{A}^{+}=\mathrm{A}+\mathrm{A}^{2+}$

$6.2 \times 10^{-4}$

$4.2 \times 10^{4}$

$6.8 \times 10^{7}$

$6.9 \times 10^{-4}$

$4.2 \times 10^{4}$

$6.1 \times 10^{7}$

$D_{\mathrm{A}, \mathrm{A}+, \mathrm{A} 2+} ; D_{\mathrm{AA} 2+} / \mathrm{cm}^{2} \mathrm{~s}^{-1}$

$8.67 \times 10^{-6} ; 5.20 \times 10^{-6}$

$8.90 \times 10^{-6} ; 5.40 \times 10^{-6}$

\footnotetext{
${ }^{\mathrm{b}}$ Slow decompostion of dication improved fits.
} 
Table S4. Experimental conditions and simulation parameters for 3,3',5,5'-tetramethylbenzidine, 1d, in acetonitrile. ${ }^{\text {a }}$

\begin{tabular}{lcccccc}
\hline Concentration/ Temp. ${ }^{\circ} \mathrm{C}$ & \multicolumn{2}{c}{$1.03 \mathrm{mM} \mathbf{1 d} / 25{ }^{\circ} \mathrm{C}$} & \multicolumn{3}{c}{$3.72 \mathrm{mM} \mathbf{1 d} / 25{ }^{\circ} \mathrm{C}$} \\
\hline Electrochemical reactions & $E^{\circ}$ & $\alpha$ & $k_{s}$ & $E^{\circ}$ & $\alpha$ & $k_{s}$ \\
\hline $\mathrm{A}^{+}+\mathrm{e}=\mathrm{A}$ & 0.080 & 0.50 & 0.35 & 0.080 & 0.50 & 0.36 \\
$\mathrm{~A}^{2+}+\mathrm{e}=\mathrm{A}^{+}$ & 0.254 & 0.69 & 0.35 & 0.253 & 0.69 & 0.36 \\
\hline Chemical reactions $^{+}$ & $K$ & $k_{f}$ & $k_{b}$ & $K$ & $k_{f}$ & $k_{b}$ \\
\hline $\mathrm{A}^{+}+\mathrm{A}^{+}=\mathrm{AA}^{2+}$ & $5.4 \times 10^{3}$ & $8.0 \times 10^{8}$ & $1.5 \times 10^{5}$ & $5.4 \times 10^{3}$ & $8.0 \times 10^{8}$ & $1.5 \times 10^{5}$ \\
$\mathrm{~A}^{+}+\mathrm{A}^{+}=\mathrm{A}+\mathrm{A}^{2+}$ & $1.1 \times 10^{-3}$ & $4.0 \times 10^{2}$ & $3.5 \times 10^{5}$ & $1.2 \times 10^{-3}$ & $4.0 \times 10^{2}$ & $3.4 \times 10^{5}$ \\
$\mathrm{~A}^{2+}=\mathrm{Prod}^{\mathrm{b}}$ & $1.0 \times 10^{9}$ & 0.17 & $1.7 \times 10^{-10}$ & $1.0 \times 10^{9}$ & 0.17 & $1.7 \times 10^{-10}$ \\
$D_{\mathrm{A}} ; D_{\mathrm{A}+, \mathrm{A} 2+, \mathrm{AA} 2+} / \mathrm{cm}^{2} \mathrm{~s}^{-1}$ & \multicolumn{7}{c}{$1.44 \times 10^{-5} ; 1.00 \times 10^{-5}$} & & $1.42 \times 10^{-5} ; 1.00 \times 10^{-5}$ \\
\hline
\end{tabular}

Concentration/ Temp. ${ }^{\circ} \mathrm{C} \quad 1.18 \mathrm{mM} 1 \mathbf{d} / 0{ }^{\circ} \mathrm{C}$

$2.84 \mathrm{mM} 1 \mathrm{~d} / 0{ }^{\circ} \mathrm{C}$

\begin{tabular}{lcccccc} 
Electrochemical reactions & $E^{\circ}$ & $\alpha$ & $k_{s}$ & $E^{\circ}$ & $\alpha$ & $k_{s}$ \\
\hline $\mathrm{A}^{+}+\mathrm{e}=\mathrm{A}$ & 0.047 & 0.50 & 0.24 & 0.047 & 0.50 & 0.24 \\
$\mathrm{~A}^{2+}+\mathrm{e}=\mathrm{A}^{+}$ & 0.251 & 0.60 & 0.24 & 0.251 & 0.60 & 0.24 \\
\hline Chemical reactions & $K$ & $k_{f}$ & $k_{b}$ & $K$ & $k_{f}$ & $k_{b}$ \\
\hline $\mathrm{A}^{+}+\mathrm{A}^{+}=\mathrm{AA}^{2+}$ & $1.2 \times 10^{4}$ & $2.8 \times 10^{9}$ & $2.4 \times 10^{5}$ & $1.2 \times 10^{4}$ & $2.8 \times 10^{9}$ & $2.4 \times 10^{5}$ \\
$\mathrm{~A}^{+}+\mathrm{A}^{+}=\mathrm{A}+\mathrm{A}^{2+}$ & $1.7 \times 10^{-4}$ & $1.5 \times 10^{2}$ & $8.8 \times 10^{5}$ & $1.7 \times 10^{-4}$ & $1.5 \times 10^{2}$ & $8.8 \times 10^{5}$ \\
$\mathrm{~A}^{2+}=\mathrm{Prod}^{\mathrm{b}}$ & $1.0 \times 10^{9}$ & 0.20 & $2.0 \times 10^{-10}$ & $1.0 \times 10^{9}$ & 0.20 & $2.0 \times 10^{-10}$ \\
$D_{\mathrm{A}, \mathrm{A}+, \mathrm{A} 2+} ; D_{\mathrm{AA} 2+} / \mathrm{cm}^{2} \mathrm{~s}^{-1}$ & \multicolumn{7}{c}{$1.05 \times 10^{-5} ; 6.00 \times 10^{-6}$} & & $1.05 \times 10^{-5} ; 6.00 \times 10^{-6}$ \\
\hline
\end{tabular}

$\begin{array}{lll}\text { Concentration/ Temp. }{ }^{\circ} \mathrm{C} & 1.30 \mathrm{mM} \mathrm{1d} /-18{ }^{\circ} \mathrm{C} & 2.85 \mathrm{mM} \mathrm{1d} /-18{ }^{\circ} \mathrm{C}\end{array}$

\begin{tabular}{lcccccc}
\hline Electrochemical reactions & $E^{\circ}$ & $\alpha$ & $k_{s}$ & $E^{\circ}$ & $\alpha$ & $k_{s}$ \\
\hline $\mathrm{A}^{+}+\mathrm{e}=\mathrm{A}$ & 0.022 & 0.50 & 0.08 & 0.020 & 0.50 & 0.09 \\
$\mathrm{~A}^{2+}+\mathrm{e}=\mathrm{A}^{+}$ & 0.244 & 0.60 & 0.12 & 0.243 & 0.60 & 0.13 \\
\hline Chemical reactions & $K$ & $k_{f}$ & $k_{b}$ & $K$ & $k_{f}$ & $k_{b}$ \\
\hline $\mathrm{A}^{+}+\mathrm{A}^{+}=\mathrm{AA}^{2+}$ & $3.2 \times 10^{4}$ & $3.0 \times 10^{9}$ & $9.3 \times 10^{4}$ & $3.2 \times 10^{4}$ & $3.0 \times 10^{9}$ & $9.3 \times 10^{4}$ \\
$\mathrm{~A}^{+}+\mathrm{A}^{+}=\mathrm{A}+\mathrm{A}^{2+}$ & $4.2 \times 10^{-5}$ & 33 & $7.9 \times 10^{5}$ & $3.9 \times 10^{-5}$ & 33 & $8.5 \times 10^{5}$ \\
$D_{\mathrm{A}, \mathrm{A}+\mathrm{A} 2+} ; D_{\mathrm{AA} 2+} / \mathrm{cm}^{2} \mathrm{~s}^{-1}$ & \multicolumn{7}{c}{$7.10 \times 10^{-6} ; 3.90 \times 10^{-6}$} & & $7.10 \times 10^{-6} ; 3.72 \times 10^{-6}$ \\
\hline
\end{tabular}

\footnotetext{
${ }^{\mathrm{b}}$ Slow decompostion of dication improved fits.
} 
Table S5. Experimental conditions and simulation parameters for $N, N, N^{\prime}, N^{\prime}$-tetramethylbenzidine, $\mathbf{1} \mathbf{j}$, in acetonitrile. ${ }^{\text {a }}$

\begin{tabular}{lcccccc}
\hline Concentration/ Temp. ${ }^{\circ} \mathrm{C}$ & \multicolumn{2}{c}{$0.80 \mathrm{mM} \mathrm{1j} / 25^{\circ} \mathrm{C}$} & \multicolumn{3}{c}{$3.60 \mathrm{mM} \mathbf{~ j} / 25{ }^{\circ} \mathrm{C}$} \\
\hline Electrochemical reactions & $E^{\circ}$ & $\alpha$ & $k_{s}$ & $E^{\circ}$ & $\alpha$ & $k_{s}$ \\
\hline $\mathrm{A}^{+}+\mathrm{e}=\mathrm{A}$ & 0.039 & 0.50 & 0.50 & 0.032 & 0.50 & 0.50 \\
$\mathrm{~A}^{2+}+\mathrm{e}=\mathrm{A}^{+}$ & 0.225 & 0.50 & 0.37 & 0.230 & 0.50 & 0.37 \\
\hline Chemical reactions & $K$ & $k_{f}$ & $k_{b}$ & $K$ & $k_{f}$ & $k_{b}$ \\
\hline $\mathrm{A}^{+}+\mathrm{A}^{+}=\mathrm{A}+\mathrm{A}^{2+}$ & $7.3 \times 10^{-4}$ & $4.0 \times 10^{2}$ & $5.5 \times 10^{5}$ & $4.4 \times 10^{-4}$ & $4.0 \times 10^{2}$ & $9.0 \times 10^{5}$ \\
$\mathrm{~A}^{2+}=\mathrm{Prod}^{\mathrm{b}}$ & $1.0 \times 10^{9}$ & 0.13 & $1.3 \times 10^{-10}$ & $1.0 \times 10^{9}$ & 0.19 & $1.9 \times 10^{-10}$ \\
$D_{\mathrm{A}} ; D_{\mathrm{A}+} ; D_{\mathrm{A} 2+} / \mathrm{cm}^{2} \mathrm{~s}^{-1}$ & $1.50 \times 10^{-5} ; 1.10 \times 10^{-5} ; 8.30 \times 10^{-6}$ & $1.65 \times 10^{-5} ; 1.16 \times 10^{-5} ; 8.50 \times 10^{-6}$ \\
\hline
\end{tabular}

\begin{tabular}{|c|c|c|c|c|c|c|}
\hline Concentration/ Temp. ${ }^{\circ} \mathrm{C}$ & \multicolumn{3}{|c|}{$1.22 \mathrm{mM} \mathbf{1 j} / 0{ }^{\circ} \mathrm{C}$} & \multicolumn{3}{|c|}{$3.05 \mathrm{mM} \mathrm{1j} / 0{ }^{\circ} \mathrm{C}$} \\
\hline Electrochemical reactions & $E^{\circ}$ & $\alpha$ & $k_{s}$ & $E^{\circ}$ & $\alpha$ & $k_{s}$ \\
\hline $\mathrm{A}^{+}+\mathrm{e}=\mathrm{A}$ & 0.030 & 0.50 & 0.38 & 0.025 & 0.50 & 0.38 \\
\hline $\mathrm{A}^{2+}+\mathrm{e}=\mathrm{A}^{+}$ & 0.212 & 0.70 & 0.20 & 0.212 & 0.70 & 0.19 \\
\hline Chemical reactions & $K$ & $k_{f}$ & $k_{b}$ & $K$ & $k_{f}$ & $k_{b}$ \\
\hline $\mathrm{A}^{+}+\mathrm{A}^{+}=\mathrm{AA}^{2+}$ & $1.3 \times 10^{2}$ & $1.9 \times 10^{8}$ & $1.5 \times 10^{6}$ & $1.3 \times 10^{2}$ & $1.9 \times 10^{8}$ & $1.5 \times 10^{6}$ \\
\hline $\mathrm{A}^{+}+\mathrm{A}^{+}=\mathrm{A}+\mathrm{A}^{2+}$ & $4.4 \times 10^{-4}$ & 40 & $9.1 \times 10^{4}$ & $3.6 \times 10^{-4}$ & 40 & $1.1 \times 10^{5}$ \\
\hline $\mathrm{A}^{2+}=\operatorname{Prod}^{\mathrm{b}}$ & $1.0 \times 10^{9}$ & 0.19 & $1.9 \times 10^{-10}$ & $1.0 \times 10^{9}$ & 0.19 & $1.9 \times 10^{-10}$ \\
\hline$D_{\mathrm{A}, \mathrm{A}+, \mathrm{A} 2+} ; D_{\mathrm{AA} 2+} / \mathrm{cm}^{2} \mathrm{~s}^{-1}$ & \multicolumn{3}{|c|}{$1.30 \times 10^{-5} ; 7.40 \times 10^{-6}$} & \multicolumn{3}{|c|}{$1.28 \times 10^{-5} ; 7.30 \times 10^{-6}$} \\
\hline
\end{tabular}

\begin{tabular}{lcccccc}
\hline Concentration/ Temp. ${ }^{\circ} \mathrm{C}$ & \multicolumn{2}{c}{$1.05 \mathrm{mM} \mathrm{1j} /-18{ }^{\circ} \mathrm{C}$} & \multicolumn{2}{c}{$3.48 \mathrm{mM} \mathbf{~ j} /-18{ }^{\circ} \mathrm{C}$} \\
\hline Electrochemical reactions & $E^{\circ}$ & $\alpha$ & $k_{s}$ & $E^{\circ}$ & $\alpha$ & $k_{s}$ \\
\hline $\mathrm{A}^{+}+\mathrm{e}=\mathrm{A}$ & 0.026 & 0.50 & 0.12 & 0.018 & 0.50 & 0.12 \\
$\mathrm{~A}^{2+}+\mathrm{e}=\mathrm{A}^{+}$ & 0.210 & 0.65 & 0.08 & 0.210 & 0.65 & 0.08 \\
\hline Chemical reactions $^{+}$ & $K$ & $k_{f}$ & $k_{b}$ & $K$ & $k_{f}$ & $k_{b}$ \\
\hline $\mathrm{A}^{+}+\mathrm{A}^{+}=\mathrm{AA}^{2+}$ & $2.1 \times 10^{2}$ & $4.4 \times 10^{8}$ & $2.1 \times 10^{6}$ & $2.1 \times 10^{2}$ & $4.4 \times 10^{8}$ & $2.1 \times 10^{6}$ \\
$\mathrm{~A}^{+}+\mathrm{A}^{+}=\mathrm{A}+\mathrm{A}^{2+}$ & $2.2 \times 10^{-4}$ & 12 & $5.4 \times 10^{4}$ & $1.7 \times 10^{-4}$ & 12 & $7.1 \times 10^{4}$ \\
$\mathrm{~A}^{2+}=\mathrm{Prod}^{\mathrm{b}}$ & $1.0 \times 10^{9}$ & 0.27 & $2.7 \times 10^{-10}$ & $1.0 \times 10^{9}$ & 0.27 & $2.7 \times 10^{-10}$ \\
$D_{\mathrm{A}, \mathrm{A}+, \mathrm{A} 2+} ; D_{\mathrm{AA} 2+} / \mathrm{cm}^{2} \mathrm{~s}^{-1}$ & \multicolumn{2}{c}{$9.90 \times 10^{-6} ; 6.00 \times 10^{-6}$} & & $9.90 \times 10^{-6} ; 6.00 \times 10^{-6}$ \\
\hline
\end{tabular}

\footnotetext{
${ }^{\mathrm{b}}$ Slow decompostion of dication improved fits.
} 
Table S6. Experimental conditions and simulation parameters for 4,4'-bis(dimethylamino)-3,3'dimethoxybiphenyl, 1e, in acetonitrile. ${ }^{\mathrm{a}}$

\begin{tabular}{|c|c|c|c|c|c|c|}
\hline Concentration/ Temp. ${ }^{\circ} \mathrm{C}$ & \multicolumn{3}{|c|}{$2.02 \mathrm{mM} \mathrm{1e} / 25^{\circ} \mathrm{C}$} & \multicolumn{3}{|c|}{$4.06 \mathrm{mM} 1 \mathrm{e} / 25^{\circ} \mathrm{C}$} \\
\hline Electrochemical reactions & $E^{\circ}$ & $\alpha$ & $k_{s}$ & $E^{\circ}$ & $\alpha$ & $k_{s}$ \\
\hline $\mathrm{A}^{+}+\mathrm{e}=\mathrm{A}$ & 0.121 & 0.5 & 0.10 & 0.127 & 0.5 & 0.13 \\
\hline $\mathrm{A}^{2+}+\mathrm{e}=\mathrm{A}^{+}$ & 0.122 & 0.6 & 0.07 & 0.124 & 0.5 & 0.05 \\
\hline Chemical reactions & $K$ & $k_{f}$ & $k_{b}$ & $K$ & $k_{f}$ & $k_{b}$ \\
\hline $\mathrm{A}^{+}+\mathrm{A}^{+}=\mathrm{AA}^{2+\mathrm{b}}$ & $1.0 \times 10^{9}$ & $2.8 \times 10^{3}$ & $2.8 \times 10^{-6}$ & $1.0 \times 10^{9}$ & $2.8 \times 10^{3}$ & $2.8 \times 10^{-6}$ \\
\hline $\mathrm{A}^{+}+\mathrm{A}^{+}=\mathrm{A}+\mathrm{A}^{2+}$ & 0.96 & 82 & 85 & 1.1 & 82 & 72 \\
\hline$D_{\mathrm{A}, \mathrm{A}+, \mathrm{A} 2+, \mathrm{AA} 2+} / \mathrm{cm}^{2} \mathrm{~s}^{-1}$ & \multicolumn{3}{|c|}{$1.47 \times 10^{-5}$} & \multicolumn{3}{|c|}{$1.59 \times 10^{-5}$} \\
\hline
\end{tabular}

$\mathrm{b}$ Inclusion of a slow, irreversible dimerization reaction improved the fits as low scan rates.

Table S7. Experimental conditions and simulation parameters for 4,4'-bis(diethylamino)-3,3'dimethoxybiphenyl, 1f, in acetonitrile. ${ }^{\mathrm{a}}$

Concentration/ Temp. ${ }^{\circ} \mathrm{C} \quad 1.95 \mathrm{mM}$ 1f $/ 25^{\circ} \mathrm{C} \quad 3.94 \mathrm{mM} \mathrm{1f} / 25^{\circ} \mathrm{C}$

\begin{tabular}{lcccccc}
\hline Electrochemical reactions & $E^{\circ}$ & $\alpha$ & $k_{s}$ & $E^{\circ}$ & $\alpha$ & $k_{s}$ \\
\hline $\mathrm{A}^{+}+\mathrm{e}=\mathrm{A}$ & 0.062 & 0.5 & 0.09 & 0.065 & 0.5 & 0.08 \\
$\mathrm{~A}^{2+}+\mathrm{e}=\mathrm{A}^{+}$ & 0.103 & 0.5 & 0.07 & 0.104 & 0.5 & 0.05 \\
\hline Chemical reactions & $K$ & $k_{f}$ & $k_{b}$ & $K$ & $k_{f}$ & $k_{b}$ \\
\hline $\mathrm{A}^{2+}=\mathrm{Prod}^{\mathrm{b}}$ & $1.0 \times 10^{9}$ & 0.03 & $3.0 \times 10^{-11}$ & $1.0 \times 10^{9}$ & 0.03 & $3.0 \times 10^{-11}$ \\
$D_{\mathrm{A}, \mathrm{A}+\mathrm{A} 2+} / \mathrm{cm}^{2} \mathrm{~s}^{-1}$ & & $1.23 \times 10^{-5}$ & & & $1.24 \times 10^{-5}$ & \\
\hline
\end{tabular}

${ }^{\mathrm{b}}$ Slow decompostion of dication improved fits.

Table S8. Experimental conditions and simulation parameters for $N, N, N^{\prime}, N^{\prime}, 3,3^{\prime}$-hexamethylbenzidine, 1g, in acetonitrile. ${ }^{\mathrm{a}}$

\begin{tabular}{lccc}
\hline Concentration/ Temp. ${ }^{\circ} \mathrm{C}$ & \multicolumn{3}{c}{$3.50 \mathrm{mM} 1 \mathrm{~g} / 25{ }^{\circ} \mathrm{C}$} \\
\hline Electrochemical reactions & $E^{\circ}$ & $\alpha$ & $k_{s}$ \\
\hline $\mathrm{A}^{+}+\mathrm{e}=\mathrm{A}$ & 0.253 & 0.5 & 0.13 \\
$\mathrm{~A}^{2+}+\mathrm{e}=\mathrm{A}^{+}$ & 0.208 & 0.5 & 0.13 \\
$D_{\mathrm{A}, \mathrm{A}+\mathrm{A} 2+} / \mathrm{cm}^{2} \mathrm{~s}^{-1}$ & & $1.51 \times 10^{-5}$ & \\
\hline
\end{tabular}


Table S9. Experimental conditions and simulation parameters for $N, N, N^{\prime}, N^{\prime}, 3,3^{\prime}, 5,5^{\prime}$-octamethyl benzidine, $\mathbf{1 h}$, in acetonitrile. ${ }^{\mathrm{a}}$

\begin{tabular}{lcccccc}
\hline Concentration/ Temp. ${ }^{\circ} \mathrm{C}$ & \multicolumn{2}{c}{$1.70 \mathrm{mM}$ 1h $/ 25^{\circ} \mathrm{C}$} & \multicolumn{3}{c}{$3.72 \mathrm{mM} \mathbf{1 h} / 25{ }^{\circ} \mathrm{C}$} \\
\hline Electrochemical reactions & $E^{\circ}$ & $\alpha$ & $k_{s}$ & $E^{\circ}$ & $\alpha$ & $k_{s}$ \\
\hline $\mathrm{A}^{+}+\mathrm{e}=\mathrm{A}$ & 0.393 & 0.5 & 0.13 & 0.389 & 0.5 & 0.13 \\
$\mathrm{~A}^{2+}+\mathrm{e}=\mathrm{A}^{+}$ & 0.302 & 0.5 & 0.17 & 0.308 & 0.5 & 0.17 \\
\hline Chemical reactions & $K$ & $k_{f}$ & $k_{b}$ & $K$ & $k_{f}$ & $k_{b}$ \\
\hline $\mathrm{A}^{+}+\mathrm{A}^{+}=\mathrm{AA}^{2+\mathrm{b}}$ & $1.0 \times 10^{9}$ & $7.6 \times 10^{4}$ & $7.6 \times 10^{-5}$ & $1.0 \times 10^{9}$ & $7.6 \times 10^{4}$ & $7.6 \times 10^{-5}$ \\
$D_{\mathrm{A}, \mathrm{A}+, \mathrm{A} 2+, \mathrm{AA} 2+} / \mathrm{cm}^{2} \mathrm{~s}^{-1}$ & & $1.58 \times 10^{-5}$ & & & $1.45 \times 10^{-5}$ &
\end{tabular}

$\mathrm{b}$ Inclusion of a slow, irreversible dimerization reaction improved the fits as low scan rates.

Table S10. Experimental conditions and simulation parameters for $N, N, N^{\prime}, N^{\prime}$-tetraphenylbenzidine, 1k, in acetonitrile. ${ }^{\mathrm{a}}$

\begin{tabular}{lccc}
\hline Concentration/ Temp. ${ }^{\circ} \mathrm{C}$ & \multicolumn{4}{c}{ Unknown conc. ${ }^{\mathrm{b}} \mathbf{1 k} / 25{ }^{\circ} \mathrm{C}$} \\
\hline Electrochemical reactions & $E^{\circ}$ & $\alpha$ & $k_{s}$ \\
\hline $\mathrm{A}^{+}+\mathrm{e}=\mathrm{A}$ & 0.356 & 0.5 & 0.13 \\
$\mathrm{~A}^{2+}+\mathrm{e}=\mathrm{A}^{+}$ & 0.503 & 0.5 & 0.13 \\
\hline
\end{tabular}

b Saturated solution. 
Comment on magnitudes of dimerization rate constants in Tables S1-S4. The voltammograms have been fit by a mechanism in which the dimerization reaction is regarded as very fast and reversible. When optimizing the fits of simulations to the experimental data for 1a-d, it was found that the dimerization rate constants in some cases exceeded the diffusion-controlled limit, estimated to be of the order of $10^{9} \mathrm{M}^{-1} \mathrm{~s}^{-1}$. Though the precision of estimation of these rate constants is low, we nonetheless sought to find an alternative mechanism that would not require second-order reactions with rate constants larger than the diffusion-controlled limit.

Simulations of $\mathbf{1 b}$ at $-18{ }^{\circ} \mathrm{C}$ could be adequately accounted for by addition of the following reactions: $\mathrm{A}+\mathrm{A}^{\bullet+} \rightleftharpoons \mathrm{AA}^{\bullet+} ; \mathrm{AA}^{2+}+\mathrm{e}^{-} \rightleftharpoons \mathrm{AA}^{\bullet+} ; \mathrm{AA}^{3+\bullet}+\mathrm{e} \rightleftharpoons \mathrm{AA}^{2+} ; \mathrm{A}^{\bullet+}+\mathrm{A}^{2+} \rightleftharpoons \mathrm{AA}^{3+\bullet}$. The values of the rate parameters for all second-order reactions were less than diffusion-controlled. The new species that are invoked would appear to be reasonable. For example, $\mathrm{AA}^{\bullet+}$ has been found in other studies of cation radicals (see Introduction).

This exercise illustrates that there are other mechanisms that can account for electro-initiated dimerization reactions. The remainder of the data were not simulated according to the more complex mechanism. It is best to regard the dimerization reactions that are reported in Tables S1-S4 as simply being very fast reactions.

Generic Caption for Figures S6-S21. Cyclic voltammogram (full curve) with simulations according to the mechanisms discussed in the text. Substance, concentration, scan rate and temperature indicated in each figure. 


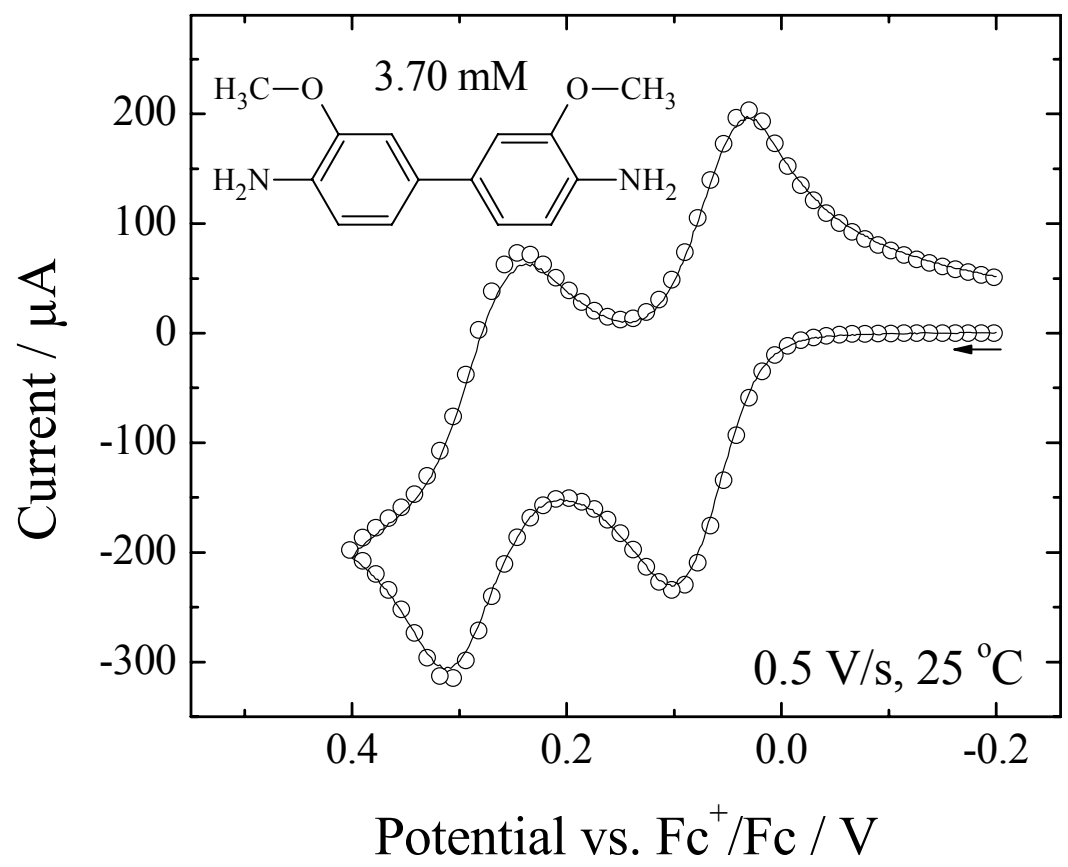

Figure S6.

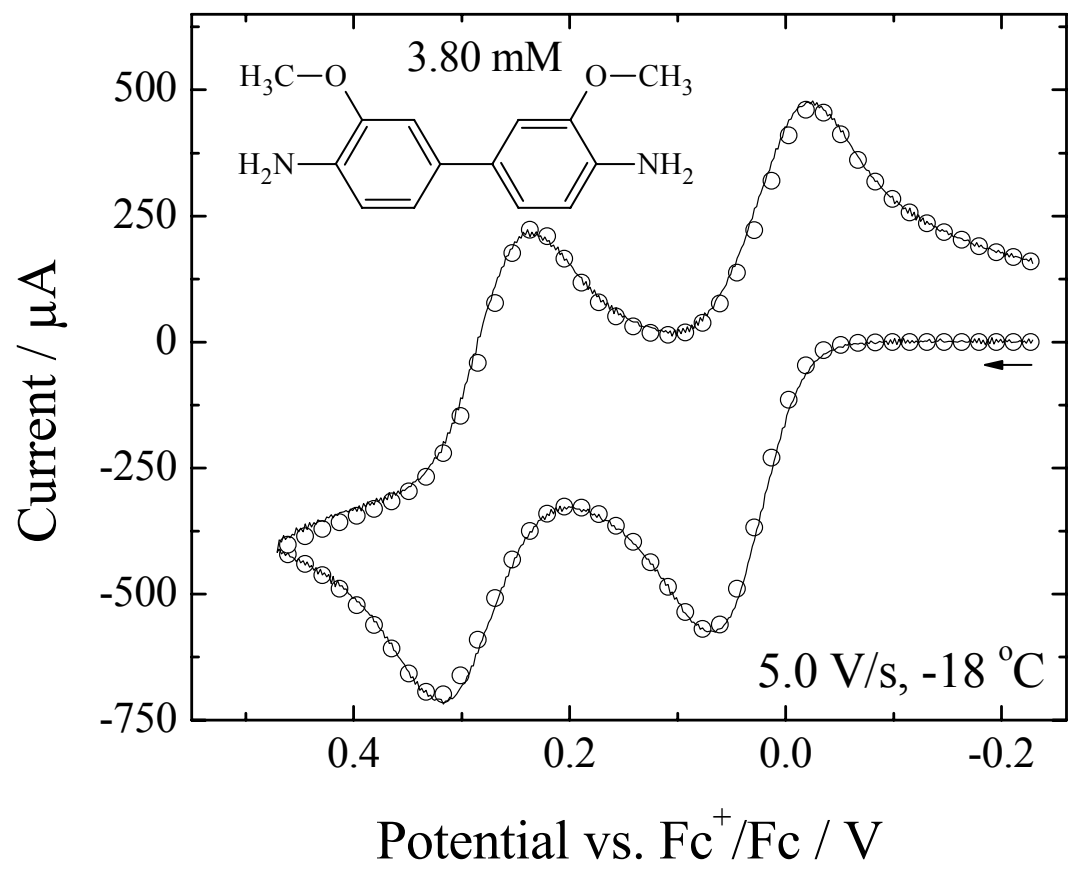

Figure S7 


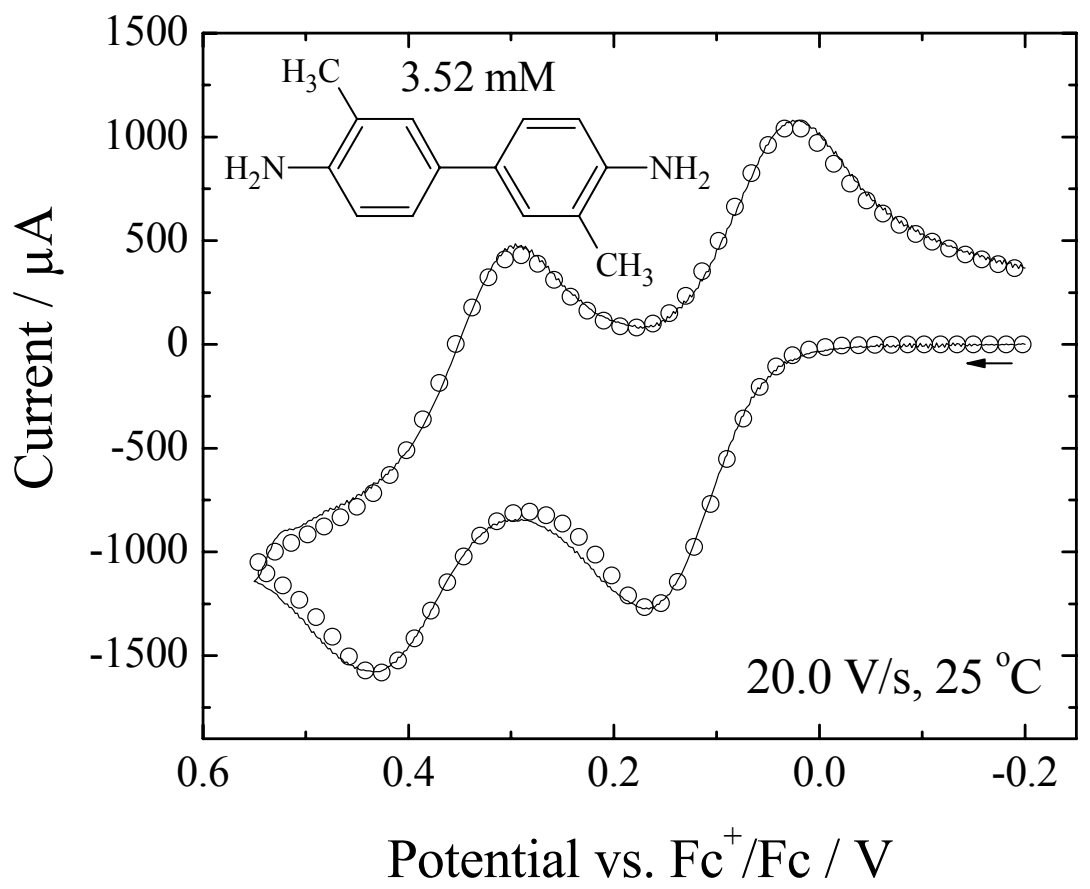

Figure S8

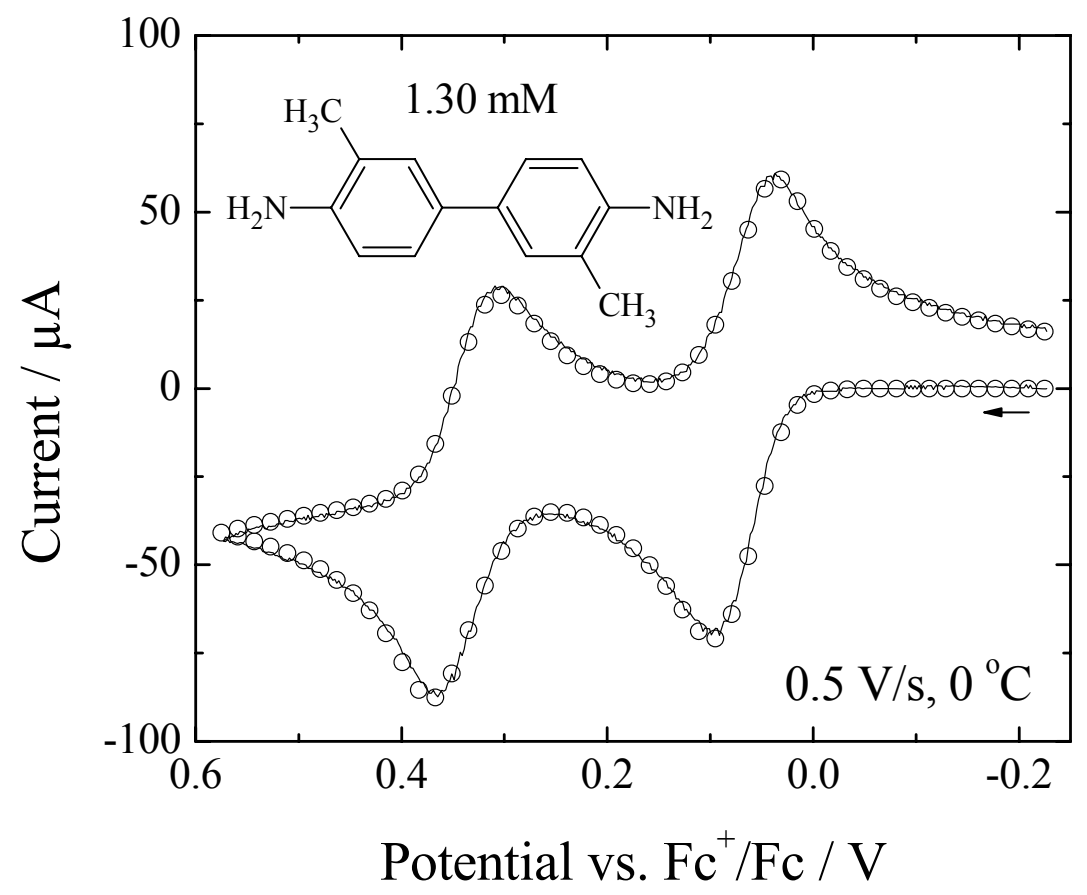

Figure S9 


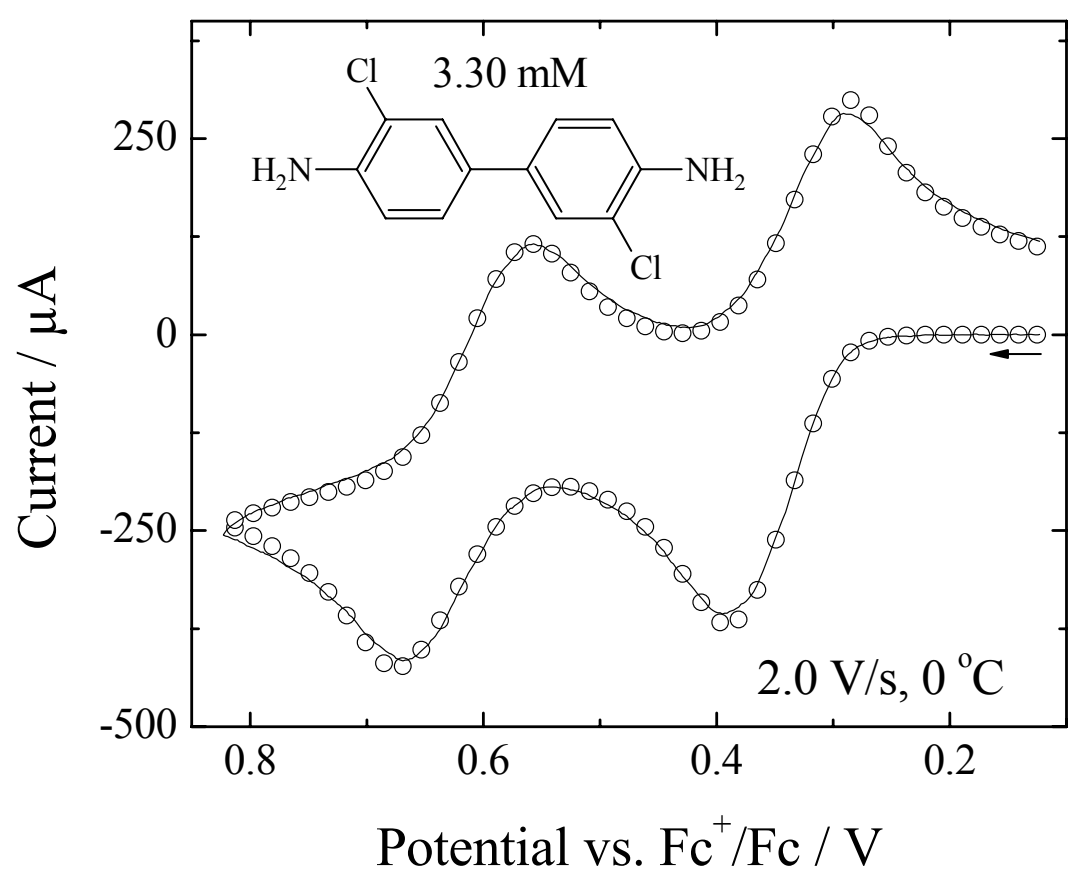

Figure S10

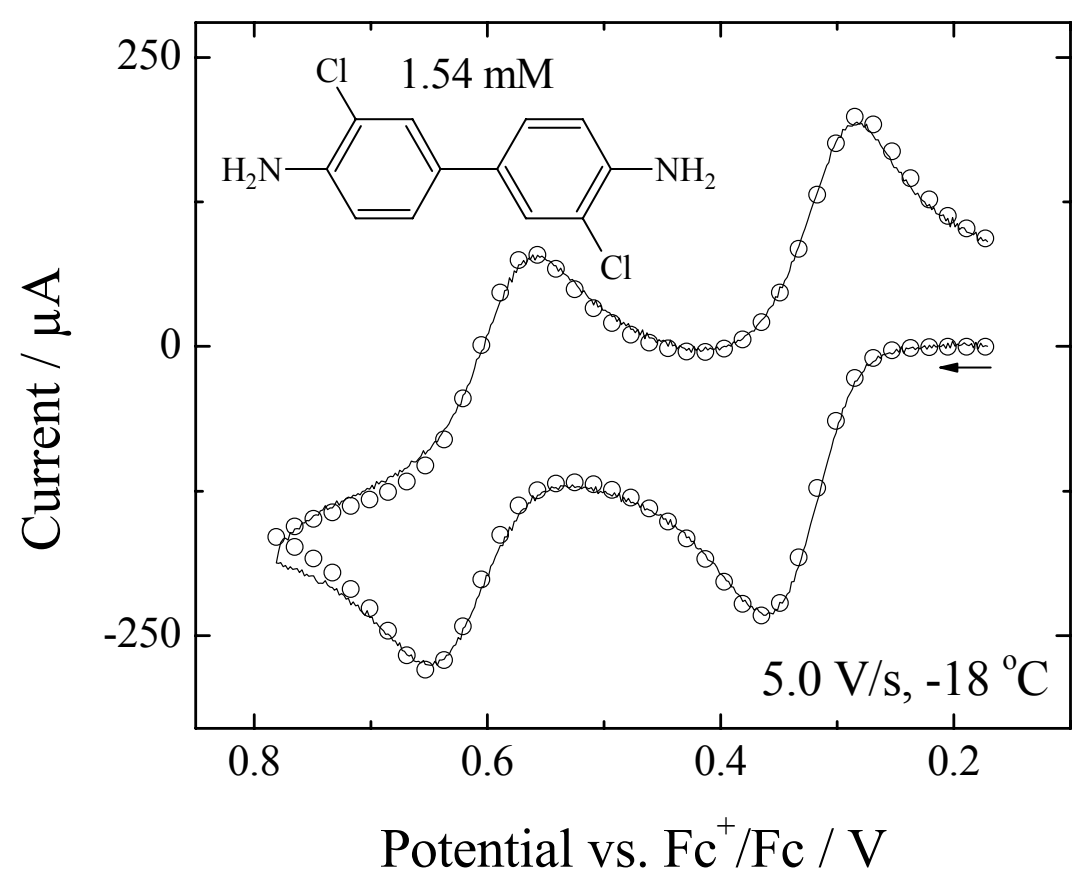

Figure S11 


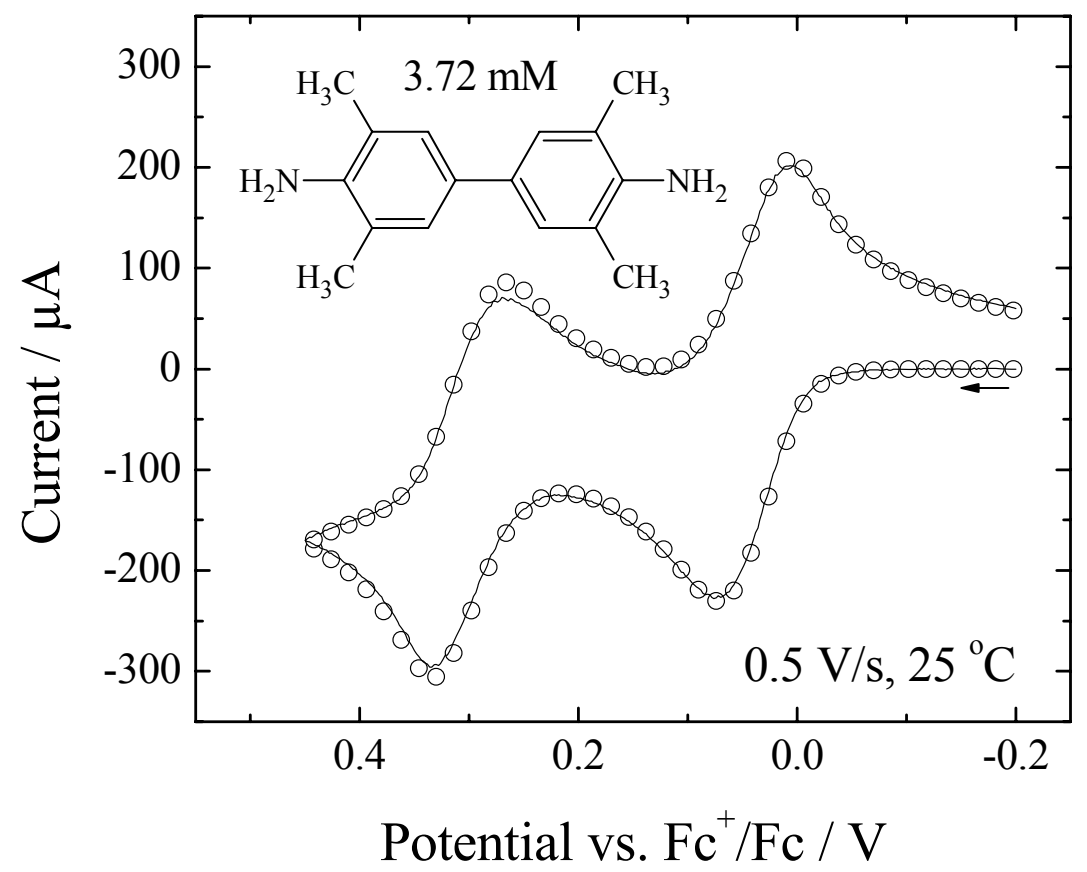

Figure S12

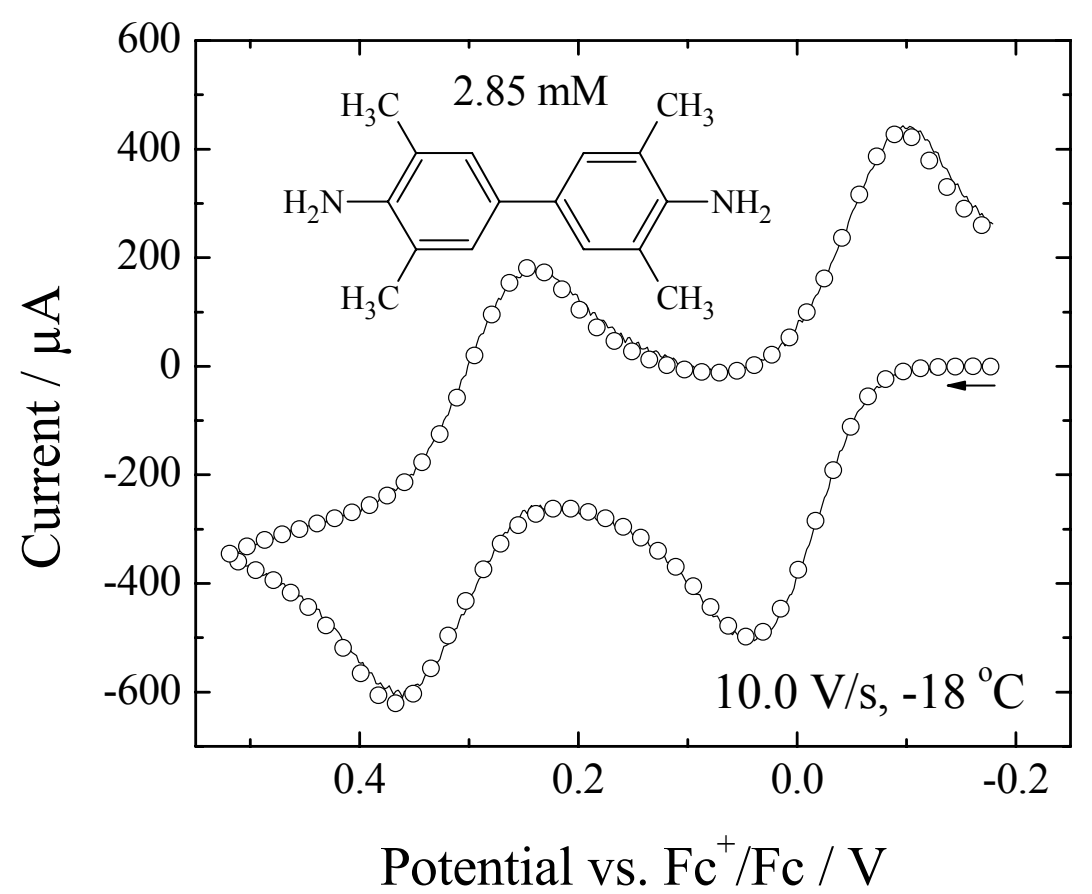

Figure S13 


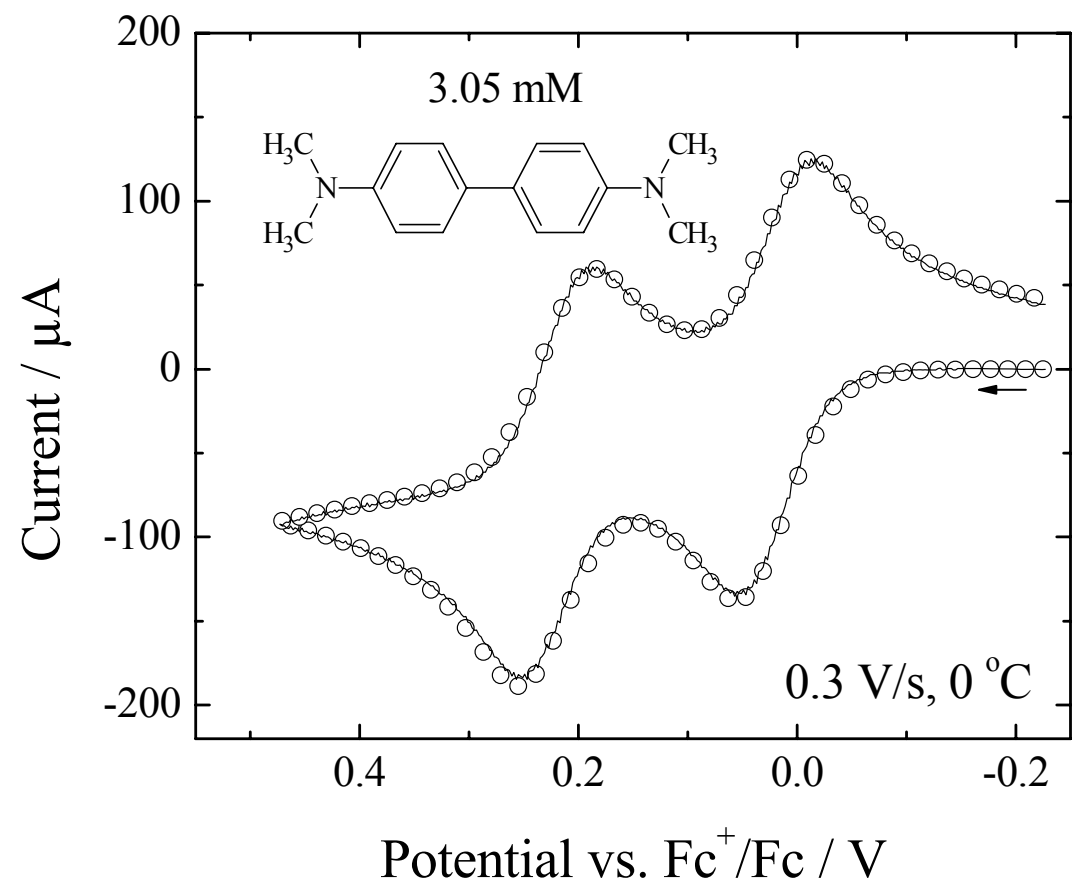

Figure S14

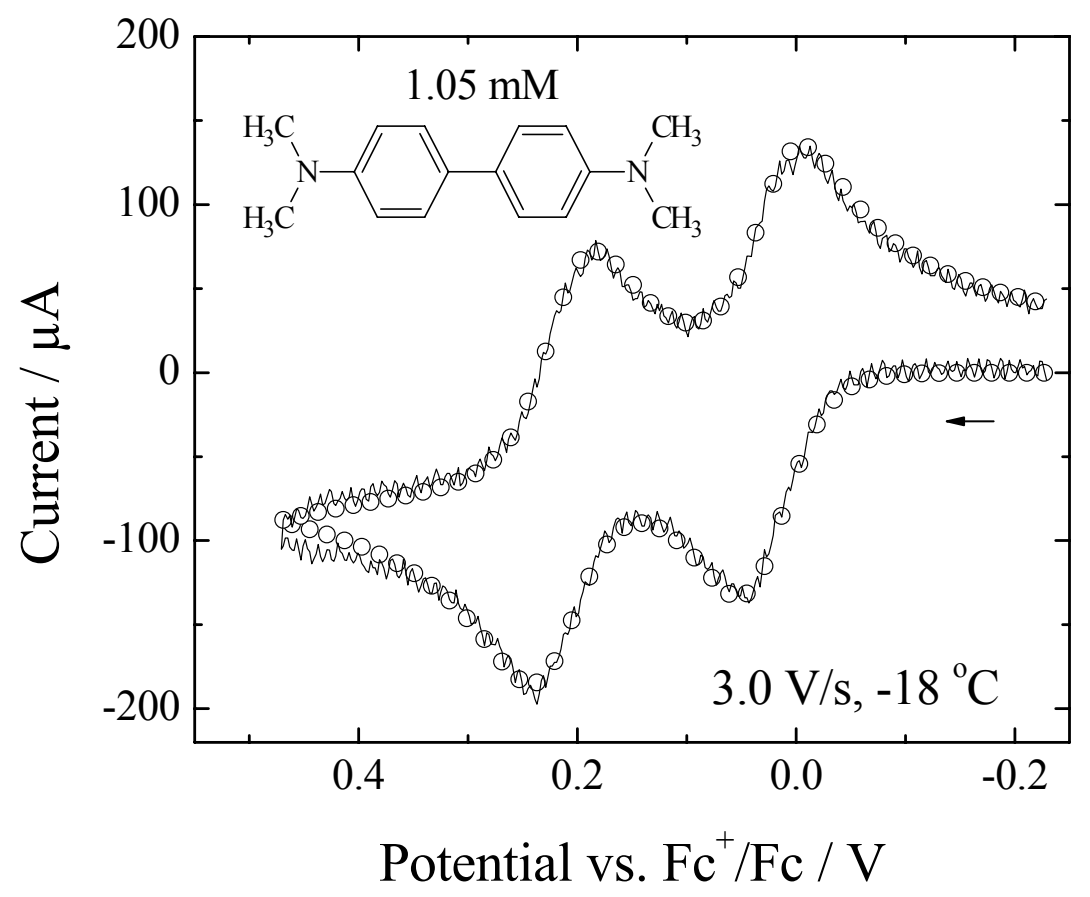

Figure S15 


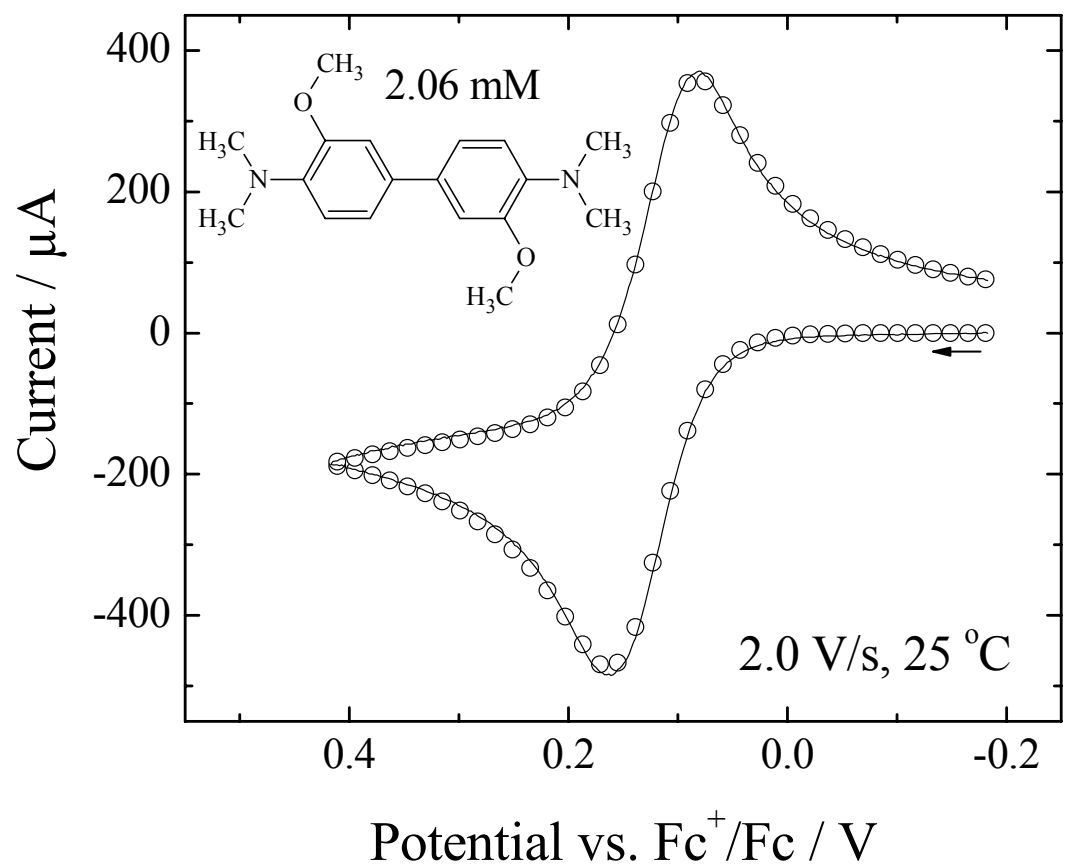

Figure S16

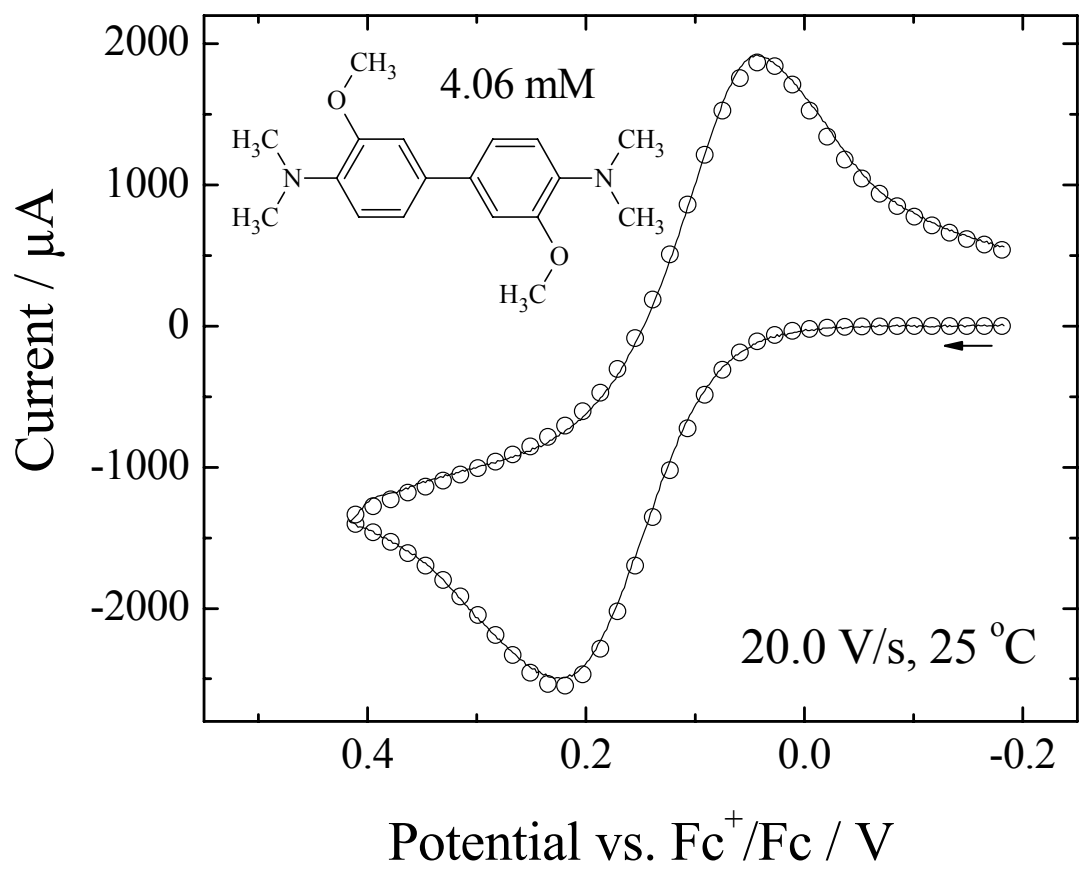

Figure S17 


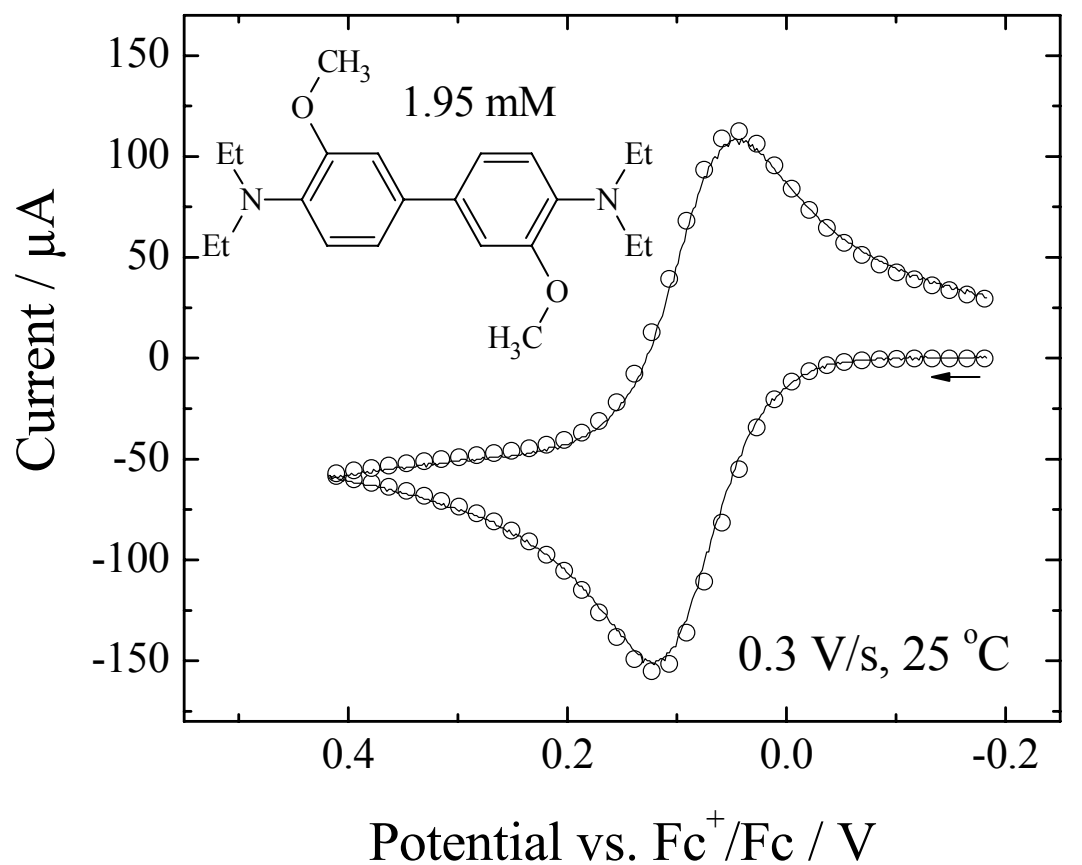

Figure S18

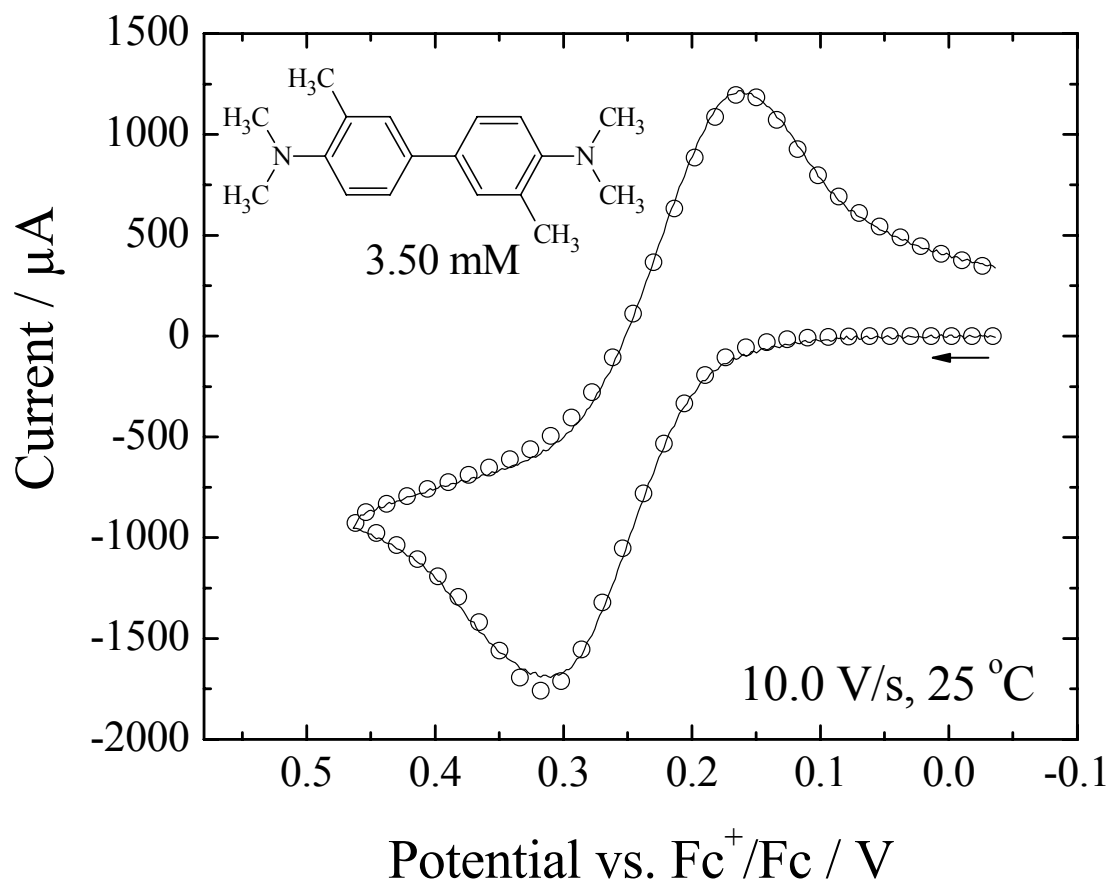

Figure S19 


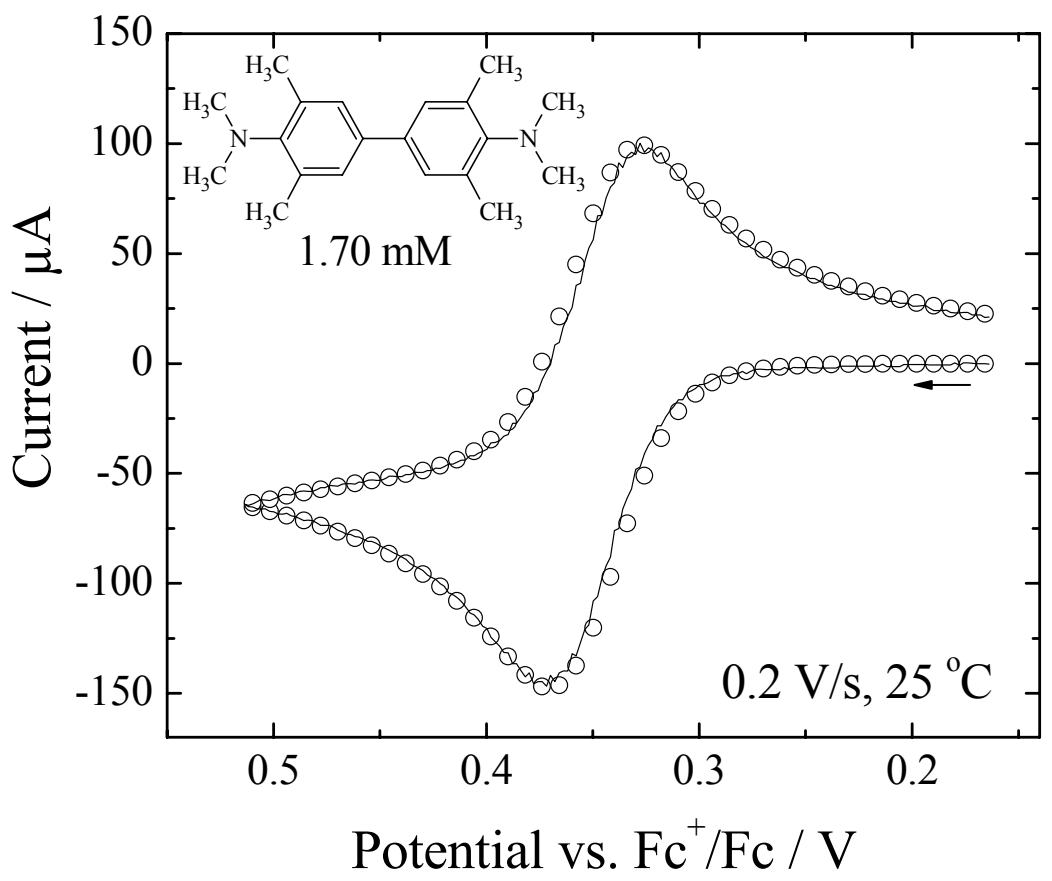

Figure S20

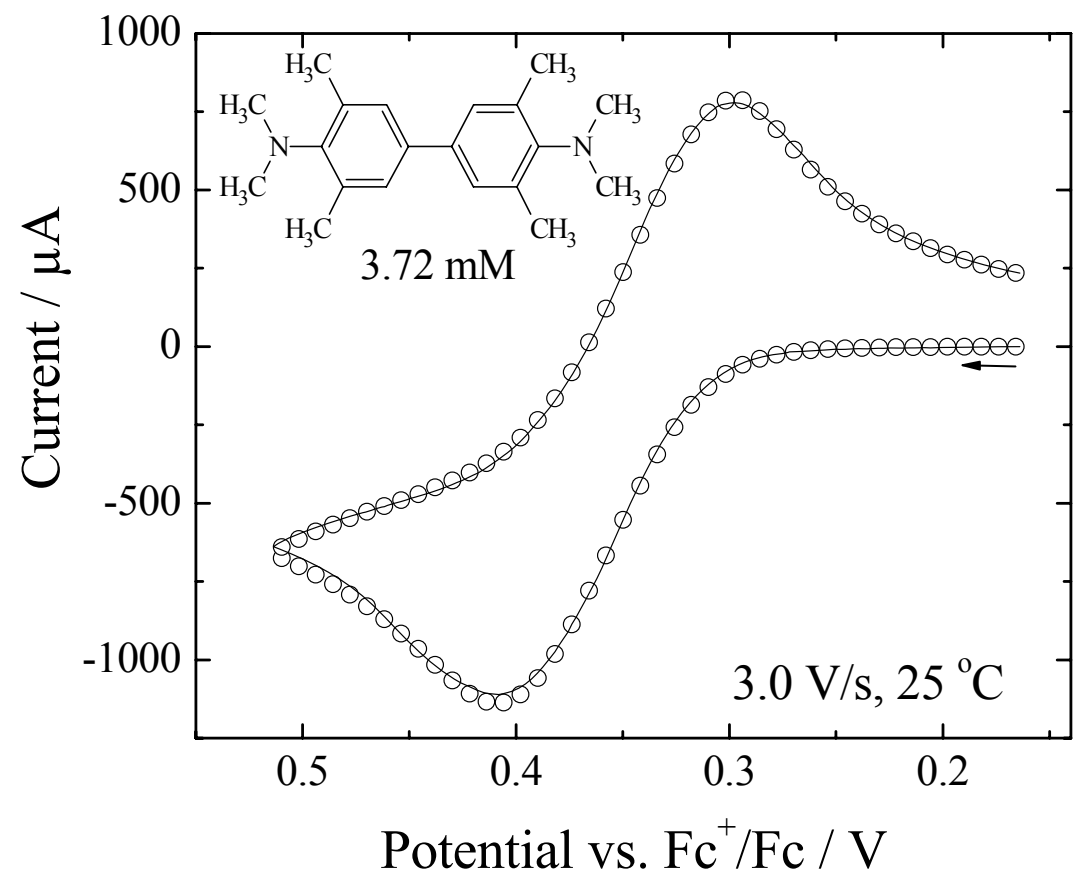

Figure S21 